LPTENS-95/20

hep-th/9505047

May 1995

\title{
HIGHER GRADING GENERALISATIONS OF THE TODA SYSTEMS'
}

\author{
Jean-Loup GERVAIS and Mikhail V. SAVELIEV² \\ Laboratoire de Physique Théorique de l'École Normale Supérieure \\ 24 rue Lhomond, 75231 Paris CÉDEX 05, France.
}

\begin{abstract}
In the present paper we obtain some integrable generalisations of the Toda system generated by flat connection forms taking values in higher $\mathbf{Z}$-grading subspaces of a simple Lie algebra, and construct their general solutions. One may think of our systems as describing some new fields of the matter type coupled to the standard Toda systems. This is of special interest in nonabelian Toda theories where the latter involve black hole target space metrics. We also give a derivation of our conformal system on the base of the Hamiltonian reduction of the WZNW model; and discuss a relation between abelian and nonabelian systems generated by a gauge transformation that maps the first grading description to the second. The latter involves grades larger than one.
\end{abstract}

\footnotetext{
${ }^{1}$ partially supported by the E.U. network "Capital Humain et Mobilité" contract \# CHRXCT920069

${ }^{2}$ On leave of absence from the Institute for High Energy Physics, 142284, Protvino, Moscow region, Russia.

${ }^{3}$ Unité Propre du Centre National de la Recherche Scientifique, associée à l'École Normale Supérieure et à l'Université de Paris-Sud.
} 


\section{Introduction}

At this time it is hardly necessary to emphasize the importance of Toda systems. In particular, much activity is devoted to the solution of affine Toda theories. Although, the coming discussion may be applied straightforwardly to this case, we will be explicitly concerned only with the non affine case, i.e. with generalizations of Toda systems associated with simple finite dimensional Lie algebras. In this connection it was shown in our paper [1], that nonabelian Toda theories provide exactly solvable conformal systems in the presence of a black holet. They correspond to gauged WZNW models with a nilpotent gauge group, and are thus basically different from the ones considered at the begining of the nineties following Witten, see e.g., [3], [4, a review [5], and references therein. The next, quite natural step, is to extend this approach so to obtain more general black matter configurations in the presence of some reasonable matter fields, in attempting to describe, in particular, black hole formation from collapsing matter and evaporation. There were a number of papers, see e.g., a recent review [6] and references therein, where such configurations have been discussed. Moreover, as it was shown in some of them, e.g., [7], [8], [9], the corresponding systems containing the conformal metric factor, the dilaton, and scalar matter fields, become solvable (in fact, of the simplest - Liouville type) for a special choice of the coupling constants. In the general context of two-dimensional exactly integrable conformal systems, the arising of configurations with a more general target space metric and additional matter fields seems to be very promising and important, and also very natural. At the same time, having an experience with a study of black holes on the base of nonabelian Toda theories, we realised that to incorporate matter fields in the game, one should deal with some relevant generalisation of these theories which possesses field components of a different nature. In a sense, the nearest, while not precise and still far-awaystanding analogue of such a picture, can be observed from a consideration of the systems associated with the subspaces $\mathcal{G}_{-1} \oplus \mathcal{G}_{-1 / 2} \oplus \mathcal{G}_{0} \oplus \mathcal{G}_{+1 / 2} \oplus \mathcal{G}_{+1}$ of a simple Lie algebra $\mathcal{G}$ endowed with a half-integral $\left(\mathbf{Z}_{2}{ }^{-}\right)$gradation, see e.g., [10], [11], [12], [13]; and from a component form of the supersymmetric Toda equations associated with a superalgebra [14]. This is why we decided to turn to a problem of constructing a generalisation of the Toda system associated with higher grading subspaces of a $\mathbf{Z}$-graded simple Lie algebra $\mathcal{G}$. The most

\footnotetext{
${ }^{4}$ For more recent developments in this direction see ref.[2].
} 
general form of two-dimensional systems generated by a flat connection with values in an arbitrary graded Lie algebra, has been discussed in [11] and references therein. In this, the consistency and integrability of the equations result from the grading condition imposed on the connection form, which implies the general solution of the system in a form of holomorphic factorisation under the condition of finiteness of the growth of the corresponding Lie algebra. However, for the systems generated by higher grading subspaces, the given formulation seems to be too abstract, see equations $(2.2)-(2.6)$, and for our aims we need to have a more precise form of the equations (desirely, maximally close to the Toda systems (2.15)) and their solutions, which takes into account the properties of a simple Lie algebra and its gradation.

The standard Toda theories are obtained by requiring that there exists a gauge, where the underlying flat connection has non zero components only in appropriate grading subspaces of a simple Lie algebra endowed with a $\mathbf{Z}$ gradation, with grades zero, one and minus one. In the present paper we give a derivation of a system which generalises the Toda theories (2.15) by some additional functions arising from the values of the flat connection form in higher grading subspaces. These new fields, as it can be seen from the given expression for the effective action, can be interpreted as matter fields coupled to the usual Toda system. There is a limit, namely a relevant specialisation the Inönü-Wigner contraction, where the back reaction of these new fields to the Toda ones goes to zero, so that they simply propagate in the fields generated by the standard Toda solutions. Our general and rather explicit systems are illustrated by examples of the simple Lie series $A_{r}$ endowed with the principal gradation, and the algebra $B_{3}$ endowed with a nonprincipal gradation. Then, using an appropriate modification of the method given in [11], which includes, in fact, some novel ingredients in comparison with the integration scheme for the Toda system, we obtain the general solution of the Goursat (boundary value) problem for our system determined by the necessary number of arbitrary functions. The expression for the solution gives a generalisation of the tau-function responsable for the Toda system. Here, following a line of [13], we give also a derivation of our conformal

\footnotetext{
${ }^{5}$ Note that for the case of the area-preserving diffeomorphisms on $T^{2}$, this project is recently realised in 15] in a form of some higher grading integrable generalisation of the continuous Toda system. It seems that these equations can be relevant for a description of matter fields with a background of the "heavenly" target space, in particular, under an appropriate contraction procedure where the matter fields do not react on the Toda fields.
} 
system on the base of the Hamiltonian reduction [12] of the WZNW model. The conformal properties of the system result applying the stress-energy tensor method of [17]. It is interesting to note that here the corresponding $W$-elements arise as decomposition coefficients of the connection form in the so-called $W$-gauge, for a definition see [13]. After that we discuss a relation between abelian and nonabelian systems. In terms of the associated $W$-algebras, our reasonings mean, in particular, that, starting from the $W$ algebra corresponding to the maximal number of the grading subspaces for the principal gradation of $\mathcal{G}$, one can obtain the $W$-algebras associated with systems for other gradations, just imposing a relevant differential polynomial constraints. In terms of the associated $W$-geometries [18], [19], it means that the target space corresponding to the system generated by the maximal number of the grading subspaces for the principal gradation of $\mathcal{G}$, contains all possible types of the metrics associated with nonabelian systems, and so all possible types of the black matter of the type under consideration here, are described by this system. As an illustration of the form of the $W$-elements for nonabelian systems, we represent examples of the 2 nd order for a gradation of the series $B_{r}$ when the subalgebra $\mathcal{G}_{0}$ is isomorphic to the sum of $r-1$ copies of $g l(1)$ and $s l(2, \mathbf{C})$ subalgebra; and briefly discuss with them the problem of the integrability of the corresponding nonabelian system on a half-line. Finally, by an example of the series $A_{r}$ endowed with the principal gradation, we obtain a Bäcklund type transformation for our system, and give a construction of the corresponding $W$-elements.

\section{Derivation of the Equations}

Let $\mathcal{M}$ be a two-dimensional manifold with local coordinates $z_{ \pm}$, and $\mathcal{G}=$ Lie $G=\oplus_{m} \mathcal{G}_{m}$ be a $\mathbf{Z}$-graded simple Lie algebra. Consider a flat connection $A$ in the trivial fibre bundle $\mathcal{M} \times G \rightarrow \mathcal{M}$ with $A_{+^{-}}$and $A_{-}$-components taking values, respectively, in the subspaces $\oplus_{m=0}^{m_{+}} \mathcal{G}_{m}$ and $\oplus_{m=0}^{m_{-}} \mathcal{G}_{-m}$ of the $\mathcal{G}$. Then the zero curvature condition written in a form

$$
\left[\partial_{+}+E_{0}^{+}+\sum_{m=1}^{m_{+}} E_{m}^{+}, \partial_{-}+E_{0}^{-}+\sum_{m=1}^{m_{-}} E_{m}^{-}\right]=0,
$$

with $\mathcal{G}$-algebra valued functions $E_{m}^{ \pm}\left(z_{+}, z_{-}\right) \in \mathcal{G}_{ \pm m}$, gives a nonlinear integrable system of partial differential equations which, for simplicity, we 
consider for $m_{+}=m_{-}=M$ and with a gauge corresponding to $E_{0}^{+}=0$. Namely,

$$
\begin{aligned}
& \partial_{+} E_{0}^{-}+\sum_{m=1}^{M}\left[E_{m}^{+}, E_{m}^{-}\right]=0 ; \\
& \partial_{+} E_{m}^{-}+\sum_{n=1}^{M-m-1}\left[E_{n}^{+}, E_{m+n}^{-}\right]+\left[E_{M-m}^{+}, E_{M}^{-}\right]=0 ; \\
& \partial_{+} E_{M}^{-}=0 ; \\
& \partial_{-} E_{m}^{+}+\left[E_{0}^{-}, E_{m}^{+}\right]+\sum_{n=1}^{M-m-1}\left[E_{n}^{-}, E_{m+n}^{+}\right]+\left[E_{M-m}^{-}, E_{M}^{+}\right]=0 ; \\
& \partial_{-} E_{M}^{+}+\left[E_{0}^{-}, E_{M}^{+}\right]=0 ;
\end{aligned}
$$

where $1 \leq m \leq M-1$. This is just the system written in [1], and we are going to represent it in a form maximally close to the Toda system.

Without loosing generality, one can take the function $E_{0}^{-}$in a form

$$
E_{0}^{-}=g_{0}^{-1} \partial_{-} g_{0}
$$

where $g_{0}\left(z_{+}, z_{-}\right)$is a function on the complex span of the Lie group $G_{0}$ corresponding to the subalgebra $\mathcal{G}_{0}$. Then, since

$$
\partial_{-} E_{m}^{+}+\left[E_{0}^{-}, E_{m}^{+}\right] \equiv g_{0}^{-1} \partial_{-}\left(g_{0} E_{m}^{+} g_{0}^{-1}\right) g_{0},
$$

let us rewrite (2.5) and (2.6) as

$$
\begin{aligned}
& \partial_{-}\left(g_{0} E_{m}^{+} g_{0}^{-1}\right)+\sum_{n=1}^{M-m-1}\left[g_{0} E_{n}^{-} g_{0}^{-1}, g_{0} E_{m+n}^{+} g_{0}^{-1}\right] \\
& +\left[g_{0} E_{M-m}^{-} g_{0}^{-1}, g_{0} E_{M}^{+} g_{0}^{-1}\right]=0 \\
& \partial_{-}\left(g_{0} E_{M}^{+} g_{0}^{-1}\right)=0 .
\end{aligned}
$$

Up to inessential transformation, equalities (2.4) and (2.8) give

$$
E_{M}^{-}=X^{-}, \quad E_{M}^{+}=g_{0}^{-1} X^{+} g_{0} ;
$$

where $X^{ \pm}$are some constant elements of the subspaces $\mathcal{G}_{ \pm M}$. With account of these relations, equations (2.2), (2.3) and (2.5) take the form

$$
\partial_{+}\left(g_{0}^{-1} \partial_{-} g_{0}\right)+\left[g_{0}^{-1} X^{+} g_{0}, X^{-}\right]+\sum_{m=1}^{M-1}\left[E_{m}^{+}, E_{m}^{-}\right]=0
$$




$$
\begin{aligned}
& \partial_{+} E_{m}^{-}+\sum_{n=1}^{M-m-1}\left[E_{n}^{+}, E_{m+n}^{-}\right]+\left[E_{M-m}^{+}, X^{-}\right]=0 \\
& \partial_{-}\left(g_{0} E_{m}^{+} g_{0}^{-1}\right)+\sum_{n=1}^{M-m-1}\left[g_{0} E_{n}^{-} g_{0}^{-1}, g_{0} E_{m+n}^{+} g_{0}^{-1}\right] \\
& +\left[g_{0} E_{M-m}^{-} g_{0}^{-1}, X^{+}\right]=0 .
\end{aligned}
$$

With the notations

$$
E_{m}^{+}=\partial_{+} W_{m}^{+}, \quad E_{m}^{-}=g_{0}^{-1} \partial_{-} W_{m}^{-} g_{0},
$$

the above written equations] can be represented as follows:

$$
\begin{aligned}
& \partial_{+}\left(g_{0}^{-1} \partial_{-} g_{0}\right)=\left[X^{-}, g_{0}^{-1} X^{+} g_{0}\right]+\sum_{m=1}^{M-1}\left[g_{0}^{-1} \partial_{-} W_{m}^{-} g_{0}, \partial_{+} W_{m}^{+}\right] \\
& \partial_{+}\left(g_{0}^{-1} \partial_{-} W_{m}^{-} g_{0}\right)=\left[X^{-}, \partial_{+} W_{M-m}^{+}\right] \\
& +\sum_{n=1}^{M-m-1}\left[g_{0}^{-1} \partial_{-} W_{m+n}^{-} g_{0}, \partial_{+} W_{n}^{+}\right] ; \\
& \partial_{-}\left(g_{0} \partial_{+} W_{m}^{+} g_{0}^{-1}\right)=\left[X^{+}, \partial_{-} W_{M-m}^{-}\right] \\
& +\sum_{n=1}^{M-m-1}\left[g_{0} \partial_{+} W_{m+n}^{+} g_{0}^{-1}, \partial_{-} W_{n}^{-}\right]
\end{aligned}
$$

$1 \leq m \leq M-1$

Looking at these relations, we see that we have two types of equations. First, (2.12) is similar to the usual Toda equation (except that $X_{ \pm}$are fixed elements in $\mathcal{G}_{ \pm M}$, instead of $\left.\mathcal{G}_{ \pm 1}\right)$, apart from the last term. Second, the other equations involve first and higher powers of the additional fields, and thus are new. One may visualise our system as describing a set of matter fields (the fields $W_{m}^{ \pm}$) coupled to Toda fields (contained in $g_{0}$ ). From this viewpoint, the last term of (2.12) may be regarded as the back reaction of the additional fields to the Toda fields. This picture is substanciated by finding a procedure that brings this back reaction to zero for part or all of the matter fields, so that they simply propagate in the field of a Toda solution.

\footnotetext{
${ }^{6} \mathrm{By}$ convention, here and in what follows derivatives act only on the first term of products, unless parentheses indicate otherwise.

${ }^{7}$ Of course the letter $W$ does not mean that these fields are the conserved $W$ generators.

${ }^{8}$ This type of theory was considered recently for the affine case in [20].
} 
This procedure is just a special type of the Inönü-Wigner contraction where elements of the simple Lie algebra $\mathcal{G}$ under consideration are multiplied by constant parameters, some of which tend to infinity (or to zero, it depends on a definition) in a consistent way. It is clear that with such a limit procedure we end up with a non-semisimple Lie algebra. In particular, one sees that if the grading subspaces $\mathcal{G}_{m}$ are rewritten as $\lambda_{m} \mathcal{G}_{m}^{\lambda}$, and then some $\lambda$ 's tend to infinity, then, due to the gradation property, $\left[\mathcal{G}_{m}, \mathcal{G}_{n}\right] \in \mathcal{G}_{m+n}$, the procedure is consistent only with a correct choice of the singular parameters sequence. Namely, one can, in particular, provide absence of the last $M-1$ terms in (2.12). Of course, there is a number of other meaningfull possibilities; some of them are discussed in section 6 . The given conclusion can be equaly reached from the expression for the effective action (3.3) presented below.

In the simplest special case when $M=1$, i.e., the connection takes values only in the local part $\mathcal{G}_{-1} \oplus \mathcal{G}_{0} \oplus \mathcal{G}_{+1}$, these equations are reduced to the Toda system 11]

$$
\partial_{+}\left(g_{0}^{-1} \partial_{-} g_{0}\right)=\left[X^{-}, g_{0}^{-1} X^{+} g_{0}\right]
$$

where, if the gradation is consistent with some $\operatorname{sl}(2, \mathbf{C})$ subalgebra of $\mathcal{G}, X^{ \pm}$ can be considered as the nilpotent elements of this subalgebra.

Next, for $M=2$ we arrive at the system

$$
\begin{aligned}
& \partial_{+}\left(g_{0}^{-1} \partial_{-} g_{0}\right)=\left[X^{-}, g_{0}^{-1} X^{+} g_{0}\right]+\left[g_{0}^{-1} \partial_{-} W_{1}^{-} g_{0}, \partial_{+} W_{1}^{+}\right] \\
& \partial_{+}\left(g_{0}^{-1} \partial_{-} W_{1}^{-} g_{0}\right)=\left[X^{-}, \partial_{+} W_{1}^{+}\right] ; \\
& \partial_{-}\left(g_{0} \partial_{+} W_{1}^{+} g_{0}^{-1}\right)=\left[X^{+}, \partial_{-} W_{1}^{-}\right]
\end{aligned}
$$

the last two equations of which can be reduced to

$$
g_{0}^{-1} \partial_{-} W_{1}^{-} g_{0}=\left[X^{-}, W_{1}^{+}\right] ; \quad g_{0} \partial_{+} W_{1}^{+} g_{0}^{-1}=\left[X^{+}, W_{1}^{-}\right] \text {. }
$$

Then, as it was mentioned in the introduction, the system (2.16), (2.19) looks very similar to those based on half-integral gradations of $\mathcal{G}$, and to the supersymmetric Toda system associated with a superalgebra, however, with a different meaning of the functions $W_{1}^{ \pm}$which are there odd functions (with anti-commuting values).

Consider one more explicit example, namely $M=3$,

$$
\begin{aligned}
& \partial_{+}\left(g_{0}^{-1} \partial_{-} g_{0}\right)=\left[X^{-}, g_{0}^{-1} X^{+} g_{0}\right] \\
& +\left[g_{0}^{-1} \partial_{-} W_{1}^{-} g_{0}, \partial_{+} W_{1}^{+}\right]+\left[g_{0}^{-1} \partial_{-} W_{2}^{-} g_{0}, \partial_{+} W_{2}^{+}\right]=0
\end{aligned}
$$




$$
\begin{aligned}
& \partial_{+}\left(g_{0}^{-1} \partial_{-} W_{1}^{-} g_{0}\right)=\left[X^{-}, \partial_{+} W_{2}^{+}\right]+\left[g_{0}^{-1} \partial_{-} W_{2}^{-} g_{0}, \partial_{+} W_{1}^{+}\right] ; \\
& \partial_{+}\left(g_{0}^{-1} \partial_{-} W_{2}^{-} g_{0}\right)=\left[X^{-}, \partial_{+} W_{1}^{+}\right] ; \\
& \partial_{-}\left(g_{0} \partial_{+} W_{1}^{+} g_{0}^{-1}\right)=\left[X^{+}, \partial_{-} W_{2}^{-}\right]+\left[g_{0} \partial_{+} W_{2}^{+} g_{0}^{-1}, \partial_{-} W_{1}^{-}\right] ; \\
& \partial_{-}\left(g_{0} \partial_{+} W_{2}^{+} g_{0}^{-1}\right)=\left[X^{+}, \partial_{-} W_{1}^{-}\right] ;
\end{aligned}
$$

where we can also remove derivatives over $z_{+}$and $z_{-}$in equations (2.22) and (2.24), respectively,

$$
g_{0}^{-1} \partial_{-} W_{2}^{-} g_{0}=\left[X^{-}, W_{1}^{+}\right] ; \quad g_{0} \partial_{+} W_{2}^{+} g_{0}^{-1}=\left[X^{+}, W_{1}^{-}\right] .
$$

and, with the help of (2.25), represent equations (2.21) and (2.23) as

$$
\begin{aligned}
& \partial_{+}\left(g_{0}^{-1} \partial_{-} W_{1}^{-} g_{0}\right)=\left[X^{-}, \partial_{+} W_{2}^{+}\right]+\left[\left[X^{-}, W_{1}^{+}\right], \partial_{+} W_{1}^{+}\right] ; \\
& \partial_{-}\left(g_{0} \partial_{+} W_{1}^{+} g_{0}^{-1}\right)=\left[X^{+}, \partial_{-} W_{2}^{-}\right]+\left[\left[X^{+}, W_{1}^{-}\right], \partial_{-} W_{1}^{-}\right] .
\end{aligned}
$$

The analogous procedure allows one to bring system (2.12) - 2.14) in a form containing multiple commutators of the functions $W^{ \pm}$(and their derivatives) with the elements $X^{\mp}$.

Let us give also another form of writing this system. Namely, introduce the functions $V_{m}^{ \pm}=g_{0}^{ \pm 1} \partial_{ \pm} W_{m}^{ \pm} g_{0}^{\mp 1}$, with which our system looks as follows:

$$
\begin{aligned}
& \partial_{+}\left(g_{0}^{-1} \partial_{-} g_{0}\right)=\left[X^{-}, g_{0}^{-1} X^{+} g_{0}\right]+\sum_{m=1}^{M-1}\left[V_{m}^{-}, g_{0}^{-1} V_{m}^{+} g_{0}\right] ; \\
& \partial_{+} V_{m}^{-}=\left[X^{-}, g_{0}^{-1} V_{M-m}^{+} g_{0}\right]+\sum_{n=1}^{M-m-1}\left[V_{m+n}^{-}, g_{0}^{-1} V_{n}^{+} g_{0}\right] ; \\
& \partial_{-} V_{m}^{+}=\left[X^{+}, g_{0} V_{M-m}^{-} g_{0}^{-1}\right]+\sum_{n=1}^{M-m-1}\left[V_{m+n}^{+}, g_{0} V_{n}^{-} g_{0}^{-1}\right] .
\end{aligned}
$$

Note that in the given derivation of the system, we did not specify any particular $\mathbf{Z}$-gradation at all. Of course, in the same way as for a particular case of the Toda system $(M=1)$, a choice of a concrete $\mathbf{Z}$-gradation allows one to represent the system in terms of equations where the unknowns are coordinates of the corresponding homogeneous space. For the most simple case - the principal gradation, the equations become essentially more explicit. Recall that for this gradation the local part $\mathcal{G}_{-1} \oplus \mathcal{G}_{0} \oplus \mathcal{G}_{+1}$ is parametrised by the Cartan and the Chevalley elements $h_{j}$ and $X_{ \pm j}, 1 \leq j \leq r$, corresponding to the simple roots $\pi_{i}, 1 \leq i \leq r$, satisfying the defining relations

$$
\left[h_{i}, h_{j}\right]=0,\left[h_{i}, X_{ \pm j}\right]= \pm k_{j i} X_{ \pm j},\left[X_{i}, X_{-j}\right]=\delta_{i j} h_{i} ;
$$


where $r$ is the rank of a simple Lie algebra $\mathcal{G}$ with the Cartan matrix $k$.

Let us illustrate the system arising for the case of the principal gradation by an example of the series $A_{r}$. Parametrise the group element

$$
g_{0}=\exp \left(-\sum_{j=1}^{r} x_{j} h_{j}\right)
$$

Then, for $M=1$ when

$$
X^{ \pm}=\sum_{j=1}^{r} \sqrt{c_{j}^{(1)}} X_{ \pm j}, c_{i}^{(1)}=2 \sum_{j=1}^{r}\left(k^{-1}\right)_{i j},
$$

we immediately come, with some evident re-notation, to the well-known abelian Toda system

$$
\partial_{+} \partial_{-} x_{i}=e^{\rho_{i}}, \quad \rho_{i} \equiv \sum_{j=1}^{r} k_{i j} x_{j} .
$$

For $M=2$, the elements $X^{ \pm}$are

$$
X^{ \pm}=\sum_{j=1}^{r-1} \sqrt{c_{j}^{(2)}} X_{ \pm(j, j+1)},
$$

with $X_{ \pm(j, j+1)}$ being the root vectors corresponding to the sum of the simple roots $\pi_{j}$ and $\pi_{j+1}$, and $c_{i}^{(2)}$ are some constants. Parametrising the functions

$$
W_{1}^{ \pm}=\sum_{j=1}^{r} f_{j}^{ \pm}\left(z_{+}, z_{-}\right) X_{ \pm j},
$$

we have the equations

$$
\begin{aligned}
& \partial_{+} \partial_{-} x_{i}=+c_{i}^{(2)} e^{\rho_{i}+\rho_{i+1}}+c_{i-1}^{(2)} e^{\rho_{i}+\rho_{i-1}}+e^{-\rho_{i}} \partial_{+} f_{i}^{+} \partial_{-} f_{i}^{-} \\
& \partial_{ \pm} f_{i}^{ \pm}=\mp e^{\rho_{i}}\left(\sqrt{c_{i-1}^{(2)}} f_{i-1}^{\mp}-\sqrt{c_{i}^{(2)}} f_{i+1}^{\mp}\right)
\end{aligned}
$$

where one should not forget that $c_{0}^{(2)}=c_{r}^{(2)}=f_{0}^{ \pm}=f_{r+1}^{ \pm} \equiv 0$, since all of them are absent in our game. Continuing the consideration, for the last step, $M=r$, where $X^{ \pm}=X_{ \pm 12 \cdots r} \equiv X_{ \pm \max }$ are the maximal and minimal roots of $A_{r}$, respectively, we introduce the notations

$$
W_{m}^{ \pm}=\sum_{\alpha \in \Delta_{m}^{+}} f_{\alpha}^{ \pm m}\left(z_{+}, z_{-}\right) X_{ \pm \alpha}
$$


where $\Delta_{m}^{+}$is the set of the positive roots corresponding to the root vectors in the subspace $\mathcal{G}_{m}$, namely

$$
\begin{aligned}
& \Delta_{1}^{+}=\left\{\pi_{1}, \cdots, \pi_{r}\right\}, \Delta_{2}^{+}=\left\{\pi_{1}+\pi_{2}, \cdots, \pi_{r-1}+\pi_{r}\right\}, \Delta_{3}^{+}=\left\{\pi_{1}+\pi_{2}+\pi_{3},\right. \\
& \left.\cdots, \pi_{r-2}+\pi_{r-1}+\pi_{r}\right\}, \cdots, \Delta_{r-1}^{+}=\left\{\pi_{1}+\cdots+\pi_{r-1}, \pi_{2}+\cdots+\pi_{r}\right\}
\end{aligned}
$$

i.e.,

$$
\Delta_{m}^{+}=\left\{\sum_{i=1}^{m} \pi_{i}, \sum_{i=2}^{m+1} \pi_{i}, \cdots, \sum_{i=r-m+1}^{r} \pi_{i}\right\} ; \quad 1 \leq m \leq r .
$$

Moreover, sometimes, to be precise, it is suitable to re-denote $X_{\alpha}, \alpha \in \Delta_{m}^{+}$, as

$$
X_{p}^{m} \equiv X_{\sum_{i=p}^{m+p-1} \pi_{i}}, 1 \leq p \leq r-m+1 ;
$$

and also put $f_{\alpha}^{ \pm m} \equiv f_{p}^{ \pm m}$.

Then the arising equations are written as

$$
\begin{aligned}
& \partial_{+} \partial_{-} x_{i}=e^{\sum_{j=1}^{r} \rho_{j}}-\delta_{i 1} \Psi_{2}^{r-1}-\delta_{i r} \Psi_{1}^{r-1} \\
& +\sum_{n=1}^{r-2} \sum_{j=i-n+1}^{i} \Psi_{j}^{n} \\
& \partial_{+}\left[e^{-\sum_{j=i}^{m+i-1}(k x)_{j}} \partial_{-} f_{i}^{-m}\right] \\
& =\delta_{r-m+1, i} \partial_{+} f_{1}^{+(r-m)}-\delta_{1, i} \partial_{+} f_{m+1}^{+(r-m)} \\
& -\sum_{n=1}^{r-m-i+1} e^{-\sum_{j=i}^{m+n+i-1}(k x)_{j}} \partial_{-} f_{i}^{-(m+n)} \partial_{+} f_{i+m}^{+n} \\
& +\sum_{n=1}^{i-1} e^{-\sum_{j=i-n}^{m+i-1}(k x)_{j}} \partial_{-} f_{i-n}^{-(m+n)} \partial_{+} f_{i-n}^{+n}, 1 \leq i \leq r-m+1 \text {; } \\
& \partial_{-}\left[e^{-\sum_{j=i}^{m+i-1}(k x)_{j}} \partial_{+} f_{i}^{+m}\right] \\
& =-\delta_{r-m+1, i} \partial_{-} f_{1}^{-(r-m)}+\delta_{1, i} \partial_{-} f_{m+1}^{-(r-m)} \\
& +\quad \sum_{n=1}^{r-m-i+1} e^{-\sum_{j=i}^{m+n+i-1}(k x)_{j}} \partial_{+} f_{i}^{+(m+n)} \partial_{-} f_{i+m}^{-n} \\
& -\quad \sum_{n=1}^{i-1} e^{-\sum_{j=i-n}^{m+i-1}(k x)_{j}} \partial_{+} f_{i-n}^{+(m+n)} \partial_{-} f_{i-n}^{-n}, 1 \leq i \leq r-m+1 .
\end{aligned}
$$


Here

$$
\Psi_{i}^{m} \equiv e^{-\sum_{s=i}^{m+i-1} \rho_{s}} \partial_{+} f_{i}^{+m} \partial_{-} f_{i}^{-m}
$$

in $(2.38)$ and (2.39) the terms on the 3rd line corresponding to $i=1, n=$ $r-m$, and the terms on the 4th line corresponding to $i=r-m+1, n=r-m$ are evidently absent. Moreover, of course, in system (2.37) appear only those functions $\Psi^{n}$ which enter decomposition (2.35), in other words

$$
\begin{aligned}
\partial_{+} \partial_{-} x_{i} & =e^{\sum_{j=1}^{r} \rho_{j}}+\Psi_{i}^{1}+\left(\Psi_{i-1}^{2}+\Psi_{i}^{2}\right) \\
& +\left(\Psi_{i-2}^{3}+\Psi_{i-1}^{3}+\Psi_{i}^{3}\right) \\
& +\cdots \\
& +\left[\left(1-\delta_{i r}\right) \Psi_{1}^{r-1}+\left(1-\delta_{i 1}\right) \Psi_{2}^{r-1}\right] .
\end{aligned}
$$

So, equations (2.37), (2.38), and (2.39) represent a system generated by the flat connection form with values in all higher grading subspaces of the Lie algebra $A_{r}$ endowed with the principal gradation; its general solution follows from the formulas given below.

Note that in the case under consideration, the subalgebra $\mathcal{G}_{0}$ contains exactly $m-1$ elements commuting with the subspace $\mathcal{G}_{m}$, namely

$$
\begin{array}{ll}
h_{i}, r-m+2 \leq i \leq m-1 ; & h_{i}+h_{m+i}, 1 \leq i \leq r-m ; \\
& h_{r-m+1}-\sum_{i=m}^{r} h_{i} ;
\end{array}
$$

and equations (2.37), (2.38) and (2.39) reflect this property.

\section{Effective Action}

It is known that the effective action for the Toda system (2.15) can be written as

$$
\mathcal{I}_{\text {eff }}^{(1)}=\mathcal{I}_{\mathrm{WZ}}\left(g_{0}\right)-\lambda \int_{\mathcal{M}} \operatorname{tr}\left(X^{-} g_{0}^{-1} X^{+} g_{0}\right),
$$

where $\mathcal{I}_{\mathrm{WZ}}\left(g_{0}\right)$ is the Wess-Zumino type action, see e.g. [12]. For system (2.16) - (2.18), i.e. for the case with $M=2$, the effective action is written in terms of the functions $g_{0}$ and $W_{1}^{ \pm}$, namely

$$
\begin{aligned}
& \mathcal{I}_{\mathrm{eff}}^{(2)}=\mathcal{I}_{\mathrm{WZ}}\left(g_{0}\right)-\lambda \int_{\mathcal{M}} \operatorname{tr}\left[\left(X^{-} g_{0}^{-1} X^{+} g_{0}\right)\right. \\
& \left.-\left(\partial_{+} W_{1}^{+} g_{0}^{-1} \partial_{-} W_{1}^{-} g_{0}\right)-\left(\left[X^{-}, W_{1}^{+}\right] \partial_{+} W_{1}^{+}\right)-\left(\left[X^{+}, W_{1}^{-}\right] \partial_{-} W_{1}^{-}\right)\right]
\end{aligned}
$$


or in terms of the functions $g_{0}$ and $V_{1}^{ \pm}$, however, with an additional integration. This form (3.2) of the action $\mathcal{I}_{\text {eff }}^{(2)}$ coincides, up to some inessential factors, with those obtained in [12] for a half-integral gradation of $\mathcal{G}$. One can see already from this first example, how it generalises the corresponding expression, e.g. in [6], including the matter fields.

In the general case under consideration here, an effective action corresponding to system (2.9) - (2.11) is written as

$$
\begin{aligned}
\mathcal{I}_{\mathrm{eff}}^{(M)} & =\mathcal{I}_{\mathrm{WZ}}\left(g_{0}\right)-\lambda \int_{\mathcal{M}} \operatorname{tr}\left[\left(X^{-} g_{0}^{-1} X^{+} g_{0}\right)\right. \\
& -\sum_{m=1}^{M-1}\left\{\left(\partial_{+} W_{m}^{+} g_{0}^{-1} \partial_{-} W_{m}^{-} g_{0}\right)+\left(\left[X^{-}, W_{m}^{+}\right] \partial_{+} W_{M-m}^{+}\right)\right. \\
& \left.+\left(\left[X^{+}, W_{m}^{-}\right] \partial_{-} W_{M-m}^{-}\right)\right\}+* * * ;
\end{aligned}
$$

where the symbol $* * *$ here means the terms of type $\left(\left[\cdots\left[X^{\mp}, W_{m_{1}}^{ \pm}\right], W_{m_{2}}^{ \pm}\right]\right.$$\cdots] \partial_{ \pm} W_{M-\sum m_{i}}^{ \pm}$.

\section{General Solution}

Let us construct the general solution to system (2.12) - (2.14) using the method given in [11]. At first, we repeat briefly the general formulation of the last reference. This introduces the necessary notations in order to proceed with our problem by specialising that method.

The flat connection $A$ in question is represented in the gradient form,

$$
A_{ \pm}=g^{-1} \partial_{ \pm} g
$$

with $g \in G$; and we take for $A_{ \pm}$the modified Gauss decomposition of $g$,

$$
g=M_{-} N_{+} g_{0-} \text { and } g=M_{+} N_{-} g_{0+},
$$

respectively. The grading conditions realised in (2.1), provide the holomorphic property of $M_{ \pm}$, namely that the functions $M_{ \pm}\left(z_{ \pm}\right) \in G_{ \pm}$satisfy the initial value problem

$$
\partial_{ \pm} M_{ \pm}\left(z_{ \pm}\right)=M_{ \pm}\left(z_{ \pm}\right) L_{ \pm}\left(z_{ \pm}\right)
$$


where

$$
L_{ \pm}\left(z_{ \pm}\right)=\sum_{m=1}^{M} \mathcal{E}_{m}^{ \pm}\left(\Phi^{ \pm}\right) ; \quad \mathcal{E}_{m}^{ \pm}\left(\Phi^{ \pm}\right)=\sum_{\alpha \in \Delta_{m}^{+}} \Phi_{\alpha}^{ \pm m}\left(z_{ \pm}\right) X_{ \pm \alpha},
$$

with arbitrary functions $\Phi_{\alpha}^{ \pm m}\left(z_{ \pm}\right)$determining the general solution to our system; the meaning of $\Delta_{m}^{+}$and $X_{ \pm \alpha}$ is the same as in (2.35). The functions $M_{ \pm}\left(z_{ \pm}\right)$can be represented by the corresponding multiplicative integrals (like the halved $S$-matrix in the quantum field theory, see e.g. [21]), or by a noncommutative version of the known exponential formula, see [22], [11].

So, with account of (4.1) and (4.2), we have

$$
A_{ \pm}=g_{0 \mp}^{-1}\left(N_{ \pm}^{-1} \partial_{ \pm} N_{ \pm}\right) g_{0 \mp}+g_{0 \mp}^{-1} \partial_{ \pm} g_{0 \mp} \cdot
$$

Differentiating the identity

$$
M_{+}^{-1} M_{-}=N_{-} \hat{g}_{0} N_{+}^{-1}
$$

with $\hat{g}_{0} \equiv g_{0+} g_{0-}^{-1}$, over $z_{ \pm}$and using equations (4.3), one can get convinced that the elements $N_{ \pm}^{-1} \partial_{ \pm} N_{ \pm}$take values in the subspaces $\oplus_{m=1}^{M} \mathcal{G}_{ \pm m}$. Rewrite now decomposition (4.5) as

$$
A_{ \pm}=g_{0 \pm}^{-1} \tilde{L}_{ \pm} g_{0 \pm}+g_{0 \mp}^{-1} \partial_{ \pm} g_{0 \mp}
$$

where

$$
\tilde{L}_{ \pm} \equiv g_{0}^{ \pm 1}\left(N_{ \pm}^{-1} \partial_{ \pm} N_{ \pm}\right) g_{0}^{\mp 1}=\sum_{m=1}^{M} \mathcal{E}_{m}^{ \pm}\left(F^{ \pm}\right)
$$

with some unknowns $F_{\alpha}^{ \pm m}\left(z_{+}, z_{-}\right)$. Note that here the functions $\tilde{L}_{ \pm}$are equal to $L_{ \pm}$only when $M=1$. However, thanks to identity (4.6), the elements $N_{ \pm}$and $\hat{g}_{0}$ are determined by the elements $M_{ \pm}\left(z_{ \pm}\right)$, and hence the functions $F^{ \pm}\left(z_{+}, z_{-}\right)$entering $\tilde{L}_{ \pm}$can be expressed in terms of the functions $\Phi_{\alpha}^{ \pm m}\left(z_{ \pm}\right)$ for an arbitrary $M$; moreover, it is clear that $F^{ \pm M}=\Phi^{ \pm M}\left(z_{ \pm}\right)$.

Now, let us accomodate this general consideration to system (2.12) (2.14), or better to (2.28) - 2.30), for which the corresponding connection components have the form

$$
A_{+}=g_{0}^{-1}\left(\sum_{m=1}^{M-1} V_{m}^{+}+X^{+}\right) g_{0}, \quad A_{-}=g_{0}^{-1} \partial_{-} g_{0}+\sum_{m=1}^{M-1} V_{m}^{-}+X^{-} .
$$


Equating decompositions (4.7) and (4.8), one has

$$
\begin{aligned}
& g_{0}^{-1}\left(\sum_{m=1}^{M-1} V_{m}^{+}+X^{+}\right) g_{0}=g_{0+}^{-1} \tilde{L}_{+} g_{0+}+g_{0-}^{-1} \partial_{+} g_{0-} \\
& g_{0}^{-1} \partial_{-} g_{0}=g_{0+}^{-1} \partial_{-} g_{0+}, \sum_{m=1}^{M-1} V_{m}^{-}+X^{-}=g_{0-}^{-1} \tilde{L}_{-} g_{0-}
\end{aligned}
$$

thereof

$$
\begin{aligned}
& g_{0}=\left(y_{0}^{+}\right)^{-1}\left(z_{+}\right) g_{0+}, \quad g_{0-}=y_{0}^{-}\left(z_{-}\right) \\
& \mathcal{E}_{M}^{-}\left(\Phi^{-}\right)=g_{0-} X^{-} g_{0-}^{-1}, \quad g_{0+}^{-1} \mathcal{E}_{M}^{+}\left(\Phi^{+}\right) g_{0+}=g_{0}^{-1} X^{+} g_{0} \\
& V_{m}^{-}=g_{0-}^{-1} \mathcal{E}_{m}^{-}\left(F^{-}\right) g_{0-}, \quad g_{0}^{-1} V_{m}^{+} g_{0}=g_{0+}^{-1} \mathcal{E}_{m}^{+}\left(F^{+}\right) g_{0+}
\end{aligned}
$$

Here $y_{0}^{ \pm} \equiv y_{0}^{ \pm}\left(z_{ \pm}\right) \in G_{0}$ are arbitrary functions of their arguments, and are expressed in terms of $\Phi^{ \pm}$. Then it is clear that $g_{0}=\left(y_{0}^{+}\left(z_{+}\right)\right)^{-1} \hat{g}_{0} y_{0}^{-}\left(z_{-}\right)$. Rewriting identity (4.6) in the form

$$
\left(y_{0}^{+}\right)^{-1} M_{+}^{-1} M_{-} y_{0}^{-}=\left[\left(y_{0}^{+}\right)^{-1} N_{-} y_{0}^{+}\right] g_{0}\left[\left(y_{0}^{-}\right)^{-1} N_{+}^{-1} y_{0}^{-}\right]
$$

one has

$$
<h\left|\left(y_{0}^{+}\right)^{-1} M_{+}^{-1} M_{-} y_{0}^{-}\right| h^{\prime}>=<h\left|g_{0}\right| h^{\prime}>
$$

where the brackets are taken between the basis vectors $\mid h>$ and the dual, $<h^{\prime} \mid$, to $\mid h^{\prime}>$, annihilated by the subspaces $\mathcal{G}_{+}$and $\mathcal{G}_{-}$, respectively. This matrix element realises a higher grading generalisation of the standard taufunction for the Toda system (2.15) depending on the necessary number of arbitrary functions $\Phi_{\alpha}^{ \pm m}\left(z_{ \pm}\right)$which determine the general solution to system $(2.12)-(2.14)$ or $(2.28)-(2.30)$. It can be rewritten as a series over the nested integrals of the products of these functions in the same way as it was done for the case of the Toda system [11] in terms of the basis $\mid h>$ with the help of the commutation relations (2.31). So, formulas (4.14), (4.11 (4.13) define the general solution to our system.

Note that expression (4.14) formally is the same as those for the Toda system (2.15), see [11]; however, the functions $L_{ \pm}$entering the initial value problem (4.3) for $M_{ \pm}$contain, in general, contributions coming from higher grading subspaces $\mathcal{G}_{m}, 1 \leq m \leq M$. The basis vectors $\mid h>$ are the highest vectors of the fundamental representations of $\mathcal{G}$ only for the case when the subalgebra $\mathcal{G}_{0}$ is abelian, i.e., for the principal gradation of $\mathcal{G}$; while for 
other $\mathbf{Z}$-gradations the subspace of the vectors annihilated by the action of $\mathcal{G}_{+}$is not already one-dimensional. At the same time, the connection form for a nonabelian Toda system (2.15) can be obtained by an appropriate gauge transformation from a connection form generated by a relevant number of higher grading subspaces of $\mathcal{G}$ endowed with the principal gradation, for which the group element $g_{0}$ is determined only by the diagonal matrix elements in (4.14) corresponding to the highest vectors of the fundamental representations of $\mathcal{G}$.

The functions $F_{m}^{ \pm}, 1 \leq m \leq m_{ \pm}-1$, entering solution (4.11) - (4.13), and in turn $\tilde{L}_{ \pm}$, are determined in terms of the matrix elements of the known element $M_{+}^{-1} M_{-}$taken between some, not necessarily highest vectors $\mid h>$ of the representation space. Consider, for example, the matrix element with a highest bra-vector $<h^{\prime} \mid$ and a ket-vector $\mid h>^{(1)}$ which is annihilated by the action of the subspaces $\mathcal{G}_{m}, m>1$. Then, differentiating the equality

$$
<h^{\prime}\left|\hat{g}_{0}^{-1} M_{+}^{-1} M_{-}\right| h>^{(1)}=<h^{\prime}\left|\left(\hat{g}_{0}^{-1} N_{-} \hat{g}_{0}\right) N_{+}^{-1}\right| h>^{(1)}=<h^{\prime}\left|N_{+}^{-1}\right| h>^{(1)},
$$

over $z_{+}$, one has a sequence of equalities

$$
\begin{aligned}
\partial_{+}<h^{\prime}\left|\hat{g}_{0}^{-1} M_{+}^{-1} M_{-}\right| h>^{(1)} & =-<h^{\prime}\left|g_{0}^{-1} \tilde{L}_{+} g_{0} N_{+}^{-1}\right| h>^{(1)}= \\
-<h^{\prime}\left|g_{0}^{-1} \mathcal{E}_{1}\left(F_{1}^{+}\right) g_{0} N_{+}^{-1}\right| h>^{(1)} & =-<h^{\prime}\left|g_{0}^{-1} \mathcal{E}_{1}\left(F_{1}^{+}\right) g_{0}\right| h>^{(1)},
\end{aligned}
$$

which determines the functions $F_{1}^{+}$. Now, knowing these functions, to find $F_{2}^{+}$we consider the matrix element with a ket-vector $\mid h>^{(2)}$ which is annihilated by the action of the subspaces $\mathcal{G}_{m}, m>2$; etc. The similar procedure allows to determine the functions $F_{m}^{-}$.

\section{Connection with WZNW model}

In this section, we show how the connection between the usual (grading one) Toda theory and WZNW model extends to our case. It seems to be important by itself, and also hopefully clarifies the derivation of the general solutions given in the above section. For background references on this aspect of the standard Toda theory, we refer to [12], while the present discussion closely follows the line of [13].

So far, the general solution was discussed with a special form (4.8), i.e.,

$$
A_{+}=g_{0}^{-1} V^{+} g_{0}, A_{-}=V^{-}+g_{0}^{-1} \partial_{-} g_{0} ; V^{ \pm} \equiv X^{ \pm}+\sum_{m=1}^{M-1} V_{m}^{ \pm} .
$$


As emphasized for instance in [13], it is useful to discuss the properties of the basic zero curvature condition

$$
\partial_{+} A_{-}-\partial_{-} A_{+}+\left[A_{+}, A_{-}\right]=0
$$

in various relevant gauges related by transformations of the type

$$
A_{ \pm} \rightarrow A_{ \pm}^{h_{0}} \equiv h_{0}^{-1} A_{ \pm} h_{0}+h_{0}^{-1} \partial_{ \pm} h_{0}
$$

where $h_{0} \in G_{0}$. From this viewpoint, the discussion was so far essentially carried out in the so-called Toda gauge where $A_{ \pm}$take the specific form displayed in (5.1). On the contrary, (4.5) is valid in any gauge, and it is easy to see that $M_{ \pm}$and $N_{ \pm}$are gauge invariant functions. In the Toda gauge, (4.5) reduces to (5.1) if we choose $g_{0 \pm}$ as in (4.11). There is another Toda gauge, where the components $A_{ \pm}$are exchanged so that the corresponding gauge potential $\tilde{A}_{ \pm}=A_{ \pm}^{g_{0}^{-1}}$ takes the form

$$
\tilde{A}_{-}=g_{0} V^{-} g_{0}^{-1}, \quad \tilde{A}_{+}=V^{+}+g_{0} \partial_{+} g_{0}^{-1} .
$$

The connection with the WZNW model is seen by changing gauge to two different choices denoted $A_{ \pm}^{(W+)}$ and $A_{ \pm}^{(W-)}$, such that $A_{+}^{(W+)}=0$ and $A_{-}^{(W-)}=$ 0 , respectively. Writing

$$
A_{ \pm}^{(W+)}=\omega_{+} A_{ \pm} \omega_{+}^{-1}+\omega_{+} \partial_{ \pm} \omega_{+}^{-1}, \quad A_{ \pm}^{(W-)}=\omega_{-}^{-1} \tilde{A}_{ \pm} \omega_{-}+\omega_{-}^{-1} \partial_{ \pm} \omega_{-},
$$

the condition just recalled implies

$$
\omega_{+}^{-1} \partial_{+} \omega_{+}=g_{0}^{-1} V^{+} g_{0}, \quad \omega_{-} \partial_{-} \omega_{-}^{-1}=g_{0} V^{-} g_{0}^{-1} .
$$

The other components give the WZNW currents according to

$$
\begin{aligned}
& A_{-}^{(W+)}=-J_{-}=\omega_{+} V^{-} \omega_{+}^{-1}+\omega_{+} g_{0}^{-1} \partial_{-}\left(g_{0} \omega_{+}^{-1}\right), \\
& A_{+}^{(W-)}=-J_{+}=\omega_{-}^{-1} V^{-} \omega_{-}+\omega_{-}^{-1} g_{0} \partial_{+}\left(g_{0}^{-1} \omega_{-}\right) .
\end{aligned}
$$

It follows from the zero curvature condition (5.2) that $\partial_{+} J_{-}=0$, and $\partial_{-} J_{+}=$ 0. Moreover, using (5.5), one verifies that

$$
J_{-}=\partial_{-} \omega \omega^{-1}, \quad J_{+}=-\omega^{-1} \partial_{+} \omega ;
$$

where

$$
\omega=\omega_{+} g_{0}^{-1} \omega_{-},
$$


so that $\omega$ is a solution of the WZNW equation. According to (4.8), $V^{ \pm} \in$ $\oplus_{m=1}^{M} \mathcal{G}_{m}$, with $V_{ \pm M}^{ \pm}=X^{ \pm}$being fixed elements in $\mathcal{G}_{ \pm M}$. Making use of relations (5.6), one easily sees that, as a consequence, $J_{-} \in \oplus_{m \geq-M} \mathcal{G}_{m}$, $J_{+} \in \oplus_{m \leq M} \mathcal{G}_{m}$, and

$$
\left.J_{-}\right|_{-M}=-X^{-},\left.\quad J_{+}\right|_{M}=-X^{+} .
$$

Those are the conditions that specify the conformal reduction from the general WZNW model, and which generalise the conditions derived in 12 and [13] for the usual (grading one) case. Next, repeating the calculation of [13], one obtains the explicit form of the WZNW solution,

$$
\begin{gathered}
\omega_{+}=q_{+}\left(z_{-}\right)\left(y_{0}^{-}\right)^{-1} N_{+} y_{0}^{-}, \quad \omega_{-}=\left(y_{0}^{+}\right)^{-1} N_{-}^{-1} y_{0}^{+} q_{-}\left(z_{+}\right) ; \\
\omega=g_{R}^{-1}\left(z_{-}\right) g_{L}^{-1}\left(z_{+}\right), \\
g_{R}=M_{-} y_{0}^{-} q_{+}^{-1}, \quad g_{L}=q_{-}^{-1}\left(y_{0}^{+}\right)^{-1} M_{+}^{-1} .
\end{gathered}
$$

Finally, (4.14) is recovered by taking matrix elements of $\omega^{-1}$ between highest weight states, and remembering definition (5.9). This gives

$$
<h\left|\omega^{-1}\right| h^{\prime}>=<h\left|g_{0}\right| h^{\prime}>=<h\left|\left(y_{0}^{+}\right)^{-1} M_{+}^{-1} M_{-} y_{0}^{-}\right| h^{\prime}>.
$$

The arbitrary functions of one variable which appear in the general solution, are contained in $L_{ \pm}$defined by (4.4). So, we have to relate $y_{0}^{ \pm}$with them. Repeating the reasonings given below (4.6), we have

$$
L_{+}=N_{-} \hat{g}_{0} N_{+}^{-1} \partial_{+} N_{+} \hat{g}_{0}^{-1} N_{-}^{-1}+N_{-} \hat{g}_{0} \partial_{+}\left(\hat{g}_{0}^{-1} N_{-}^{-1}\right) .
$$

Since $L_{+} \in \mathcal{G}_{+}$, only the first term gives nonzero contribution. Moreover, it is easily seen that, for the maximum grading component

$$
\left.L_{+}\right|_{M}=\left.\hat{g}_{0}\left(N_{+}^{-1} \partial_{+} N_{+}\right)\right|_{M} \hat{g}_{0}^{-1} .
$$

Combining (4.5) with (5.1), this gives

$$
\left.L_{+}\right|_{M}=y_{0}^{+} V^{+}\left(y_{0}^{+}\right)^{-1} .
$$

Recalling that the maximum grading component of $V^{+}$is fixed, and performing similar calculations for $L_{-}$, we arrive at the conditions

$$
\left.L_{ \pm}\right|_{ \pm M}=y_{0}^{ \pm} X^{ \pm}\left(y_{0}^{ \pm}\right)^{-1} \text {. }
$$


For the usual (grading one) case, there is only one grading component, and $y_{0 \pm}$ contain all arbitrary functions. As it was already mentioned in the previous section, this is no more true here, of course. Thus, following what we discussed in that section, we have to take matrix elements of $\omega^{-1}$ between states which are not highest weight vectors. As a result, the factors $q_{ \pm}$do not disappear, and we need to express them in terms of the arbitrary functions $\Phi_{\alpha}^{ \pm m}$. This is done as follows. Substituting expressions (5.12) in the equation for $J_{-}$, one sees that

$$
J_{-}=\partial_{-} g_{R}^{-1} g_{R}=\partial_{-} q_{+} q_{+}^{-1}+q_{+} \partial_{-}\left(y_{0}^{-}\right)^{-1} y_{0}^{-} q_{+}^{-1}-q_{+}\left(y_{0}^{-}\right)^{-1} L_{-} y_{0}^{-} q_{+}^{-1},
$$

where we made use of (4.3). It follows from (4.4) and (5.14) that we can write

$$
L_{ \pm}\left(z_{ \pm}\right)=\sum_{m=1}^{M} y_{0}^{ \pm} \tilde{\epsilon}_{m}^{ \pm}\left(z_{ \pm}\right)\left(y_{0}^{ \pm}\right)^{-1}, \quad \tilde{\epsilon}_{M}^{ \pm}=X_{ \pm}
$$

so that

$$
J_{-}=\partial_{-} q_{+} q_{+}^{-1}+q_{+} \partial_{-}\left(y_{0}^{-}\right)^{-1} y_{0}^{-} q_{+}^{-1}-\sum_{m=1}^{M} q_{+} \tilde{\epsilon}_{m}^{ \pm}\left(z_{ \pm}\right) q_{+}^{-1} .
$$

The first two terms of this relation belong to $\mathcal{G}_{+} \oplus \mathcal{G}_{0}$. One may verify that $q_{+}$can be chosen such that the current takes the form

$$
J_{-}=-X_{-}+\sum_{m \geq 0} J_{m}, \quad J_{m} \in \mathcal{G}_{m}
$$

Indeed, writing

$$
q_{+}=\exp \left(\sum_{m>0} \alpha_{m}^{+}\right), \quad \alpha_{m}^{+} \in \mathcal{G}_{m}
$$

one gets the equations

$$
\begin{aligned}
\left.J_{-}\right|_{-M} & =-X_{-}, \\
\left.J_{-}\right|_{-M+1} & =-\tilde{\epsilon}_{M-1}^{-}-\left[\alpha_{1}^{+}, X_{-}\right]=0 \\
\left.J_{-}\right|_{-M+2} & =-\tilde{\epsilon}_{M-2}^{-}-\left[\alpha_{2}^{+}, X_{-}\right]-\frac{1}{2}\left[\alpha_{1}^{+},\left[\alpha_{1}^{+}, X_{-}\right]\right]=0,
\end{aligned}
$$

and so on, which may be solved reccursively. Of course, what we are doing is just fixing gauge in the corresponding Drinfeld-Sokolov equation [23. 


\section{An Example of Nonabelian $B_{3}$ Toda System Coupled to Matter Fields}

Recall that the simplest nonabelian Toda system which describes a nontrivial target space metric, is related to the algebra $B_{2}$ endowed with a nonprincipal gradation, see [11], [24], [1]. However, the systems which incorporate a nontrivial target space metric and nontrivial matter fields, are realised for the algebras of higher rank, begining from $B_{3}$. Just this case is considered in the present section, for which we give an explicit form of the equations of motion for the Toda type and matter type fields; write down the corresponding action; and discuss, how the Inönü-Wigner contraction brings the back reaction of the matter fields to the Toda fields to zero. Consider the Lie algebra $B_{3}$ endowed with a nonprincipal gradation, namely such that

$$
\begin{aligned}
& \mathcal{G}_{0}=\left\{h_{1}, h_{2}, h_{3} ; X_{ \pm 3}\right\} ; \quad \mathcal{G}_{ \pm 1}=\left\{X_{ \pm 1}, X_{ \pm 2}, X_{ \pm 23}, X_{ \pm 233}\right\} \\
& \mathcal{G}_{ \pm 2}=\left\{X_{ \pm 12}, X_{ \pm 123}, X_{ \pm 1233}\right\} ; \quad \mathcal{G}_{ \pm 3}=\left\{X_{ \pm 12233}\right\}
\end{aligned}
$$

Then system (2.28) - (2.30) is rewritten in an explicit way in terms of the group parameters of $g_{0}\left(z_{+}, z_{-}\right)$and the functions $f_{a}^{ \pm}\left(z_{+}, z_{-}\right)$entering the decomposition $V^{ \pm}=\sum_{\alpha \in \Delta_{1}} f_{\alpha}^{ \pm} X_{ \pm \alpha}$; here and in what follows in this section, we omit the lower indices " 1 " at matter fields, and refering on system (2.28) - (2.30) suppose that there $M=2$. Furthermore, seemingly the simplest, though rather nontrivial possibility arises when one takes as $X^{ \pm}$the root vectors $X_{ \pm 1233}$; a hint for such a choice comes from the fact that just this element of $\mathcal{G}_{ \pm 2}$ commutes with the maximal number of the elements of $\mathcal{G}_{0}$, namely with $h_{1}+h_{2}$ and $2 h_{2}+h_{3}$.

Note that in what follows, to provide a possibility to make a reduction to the corresponding standard Toda system, we supply the elements $X^{ \pm}$with a constant factor $\xi$ which tends to zero in this case. Moreover, sometimes it is more suitable to use an analogous, while not completely equivalent form (2.16), (2.19) of equations (2.28) - (2.30), where the functions $v_{\alpha}^{ \pm}\left(z_{+}, z_{-}\right)$ enter the decomposition $W^{ \pm}=\sum_{\alpha \in \Delta} v_{\alpha}^{ \pm} X_{ \pm \alpha}$ with $V^{ \pm}=g_{0}^{ \pm 1} \partial_{ \pm} W^{ \pm} g_{0}^{\mp 1}$.

Let us begin with a suitable parametrisation of the function $g_{0}$. It happens that the parametrisation

$$
g_{0}=e^{a^{+} X_{+3}} \cdot e^{a^{-} X_{-3}} \cdot e^{\sum_{i=1}^{3} a_{i} h_{i}}
$$

used in [11] and then in [24], [1], is not the best one; much more relevant for 
nonabelian systems in question is the following:

$$
g_{0}=e^{\alpha h_{3}} \cdot e^{\beta\left(X_{+3}+X_{-3}\right)} \cdot e^{\gamma h_{3}} \cdot e^{\sum_{i=1}^{2} a_{i} h_{i}} .
$$

It is clear that the parameters in both of them are connected by the formulas

$$
e^{-a_{3}}=e^{-(\alpha+\gamma)} \operatorname{ch} \beta, \quad a^{+}=e^{2 \alpha} \operatorname{th} \beta, \quad a^{-}=\frac{1}{2} e^{-2 \alpha} \operatorname{sh} 2 \beta .
$$

Note also that the Wess-Zumino term $\mathcal{I}_{\mathrm{WZ}}\left(g_{0}\right)$ in parametrisation (6.2) is given by the expression

$$
\begin{aligned}
& \mathcal{I}_{\mathrm{WZ}}\left(g_{0}\right)=\frac{1}{2} \int_{\mathcal{M}}\left\{4 \partial_{+} \alpha \partial_{-} \alpha+\partial_{+}\left(a_{2}-2 \gamma\right) \partial_{-}\left(a_{2}-2 \gamma\right)\right. \\
& \left.+\partial_{+} a_{1} \partial_{-} a_{1}+\partial_{+} a_{2} \partial_{-} a_{2}+4 \partial_{+} \beta \partial_{-} \beta-4 \partial_{+}\left(a_{2}-2 \gamma\right) \partial_{-} \alpha \operatorname{ch} 2 \beta\right\} .
\end{aligned}
$$

With this parametrisation, system $(2.28)-(2.30)$ takes the form

$$
\begin{gathered}
\partial_{+} \partial_{-} a_{1}=-4 \xi e^{-a_{1}+a_{2}-2(\alpha+\gamma)} \operatorname{ch}^{2} \beta-\Phi_{1,1} \\
\partial_{+} \partial_{-} a_{2}=-4 \xi e^{-a_{1}+a_{2}-2(\alpha+\gamma)} \operatorname{ch}^{2} \beta-\Phi_{2,2}-2 \Phi_{23,23}-4 \Phi_{233,233} \\
\partial_{+}\left(\partial_{-} \gamma+\operatorname{ch} 2 \beta \partial_{-} \alpha\right)=-4 \xi e^{-a_{1}+a_{2}-2(\alpha+\gamma)} \operatorname{ch}^{2} \beta-\Phi_{23,23}-4 \Phi_{233,233} \\
\partial_{+}\left[e^{a_{2}-2 \gamma}\left(\partial_{-} \beta+\operatorname{sh} 2 \beta \partial_{-} \alpha\right)\right]=\Phi_{23,2}-2 \Phi_{233,23} \\
\partial_{+}\left[e^{-a_{2}+2 \gamma}\left(\partial_{-} \beta-\operatorname{sh} 2 \beta \partial_{-} \alpha\right)\right]=2 \xi e^{-a_{1}-2 \alpha} \operatorname{sh} 2 \beta+\Phi_{2,23}-2 \Phi_{23,233} \\
\partial_{ \pm} f_{1}^{\mp}=\mp 4 \xi F_{233}^{ \pm}, \quad \partial_{ \pm} f_{233}^{\mp}= \pm \xi F_{1}^{ \pm} \\
\partial_{ \pm} f_{2}^{\mp}=\partial_{ \pm} f_{23}^{\mp}=0 .
\end{gathered}
$$

Here, for the sake of brevity, we denote $\Phi_{\alpha, \beta} \equiv F_{\alpha}^{+} f_{\beta}^{-} ; F_{\alpha}^{+}$are the coefficients in the decomposition $g_{0}^{-1} V^{+} g_{0}=\sum_{\alpha \in \Delta} F_{\alpha}^{+} X_{+\alpha}$; the similar definitions is taken for the functions $F_{\alpha}^{-}$in terms of $f_{\alpha}^{-}$. It follows from equations (6.6) that $f_{2}^{\mp}$ and $f_{23}^{\mp}$ do not depend on $z_{ \pm}$, respectively, and, in fact, these functions can be considered as constants without loosing a generality. Note that the equations describing the corresponding standard Toda system associated with such a nonprincipal gradation of $B_{3}$, arise when $\xi$ tends to zero, $f_{2}^{ \pm}=f_{233}^{ \pm}=0$, and $f_{1}^{ \pm}, f_{23}^{ \pm}$are some nonzero fixed constants.

To emphasise better the structure of these equations, let us make a simplification, namely put $f_{2}^{ \pm}=f_{23}^{ \pm}=0$. Then system (6.4) - 6.6) is reduced 
to the following one:

$$
\begin{aligned}
& \partial_{+} \partial_{-} a_{1}=-4 \xi e^{-a_{1}+a_{2}-2(\alpha+\gamma)} \operatorname{ch}^{2} \beta-e^{-2 a_{1}+a_{2}} f_{1}^{+} f_{1}^{-}, \\
& \partial_{+} \partial_{-} a_{2}=-4 e^{-2(\alpha+\gamma)} \operatorname{ch}^{2} \beta\left[\xi e^{-a_{1}+a_{2}}+e^{a_{1}} f_{233}^{+} f_{233}^{-}\right], \\
& \partial_{+} \partial_{-} \beta=-\frac{1}{2} \operatorname{th} \beta \partial_{+} \partial_{-} a_{2}+4 \frac{\operatorname{ch} \beta}{\operatorname{sh}^{3} \beta} \partial_{+} \omega \partial_{-} \omega, \\
& \partial_{+}\left(\operatorname{cth}^{2} \beta \partial_{-} \omega\right)+\partial_{-}\left(\operatorname{cth}^{2} \beta \partial_{+} \omega\right)=\frac{1}{2} \partial_{+} \partial_{-} a_{2}, \\
& \partial_{ \pm} f_{1}^{\mp}=\mp 4 \xi e^{a_{1}-2(\alpha+\gamma)} \operatorname{ch}^{2} \beta f_{233}^{ \pm}, \\
& \partial_{ \pm} f_{233}^{\mp}= \pm \xi e^{-2 a_{1}+a_{2}} f_{1}^{ \pm} .
\end{aligned}
$$

Here we have used the new function $\omega$ defined by the relations

$$
\partial_{-} \alpha=\frac{\partial_{-} \omega}{\operatorname{sh}^{2} \beta}, \quad \partial_{+}\left(\gamma-\frac{a_{2}}{2}\right)=\frac{\partial_{+} \omega}{\operatorname{sh}^{2} \beta} ;
$$

which are noncontradictive due to equations (6.4). In the limit $\xi=0$ and $f_{\alpha}^{ \pm}=0$, these equations take the form

$$
\begin{aligned}
& \partial_{+} \partial_{-} a_{1}=\partial_{+} \partial_{-} a_{2}=0, \\
& \partial_{+} \partial_{-} \beta=4 \frac{\operatorname{ch} \beta}{\operatorname{sh}^{3} \beta} \partial_{+} \omega \partial_{-} \omega, \\
& \partial_{+}\left(\operatorname{cth}^{2} \beta \partial_{-} \omega\right)+\partial_{-}\left(\operatorname{cth}^{2} \beta \partial_{+} \omega\right)=0 ;
\end{aligned}
$$

and correspond to the following Lagrange function:

$$
\mathcal{L}=\frac{1}{2} \partial_{+} \beta \partial_{-} \beta-2 \operatorname{cth}^{2} \beta \partial_{+} \omega \partial_{-} \omega .
$$

Another simplification of system (6.7) - (6.12), similar to the last one, and effectively still without matter fields, can be obtained equating $\xi e^{-a_{1}+a_{2}}+$ $e^{a_{1}} f_{233}^{+} f_{233}^{-}=0$. Then (6.8) gives $\partial_{+} \partial_{-} a_{2}=0$; and, up to an appropriate transformation, taking $a_{2}=0$ and, for simplicity, $\xi=1$, one can choose $f_{233}^{+}=f_{233}^{-}=i e^{-a_{1}}$. Hence, from (6.12) one obtains $f_{1}^{ \pm}=\mp i \partial_{ \pm} e^{a_{1}}$, and from (6.11) it follows that $a_{1}$ satisfies the equation

$$
\partial_{+} \partial_{-} e^{a_{1}}=-4 e^{-2(\alpha+\gamma)} \operatorname{ch}^{2} \beta
$$

which coincides with (6.7) under our assumptions. The rest two equations (6.9) and (6.10) are rewritten in the standard for the corresponding nonabelian Toda equations form (6.14) and (6.15). 
As it was mentioned in section 2, to provide zero back reaction of the matter fields to the Toda fields for the systems associated with higher grading subspaces of a simple Lie algebra $\mathcal{G}$, one can apply the Inönü-Wigner contraction of $\mathcal{G}$. For the standard Toda systems such a procedure was realised in [11] and references therein; here we use it for our system (2.28) - (2.30), or, the same, for (2.16), (2.19). Multiply the elements of the subspaces $\mathcal{G}_{ \pm 1}$ and $\mathcal{G}_{ \pm 3}$ of the algebra $B_{3}$ by some parameter $\kappa^{-1}$, keeping the elements of the rest subspaces $\mathcal{G}_{0}$ and $\mathcal{G}_{ \pm 2}$ as they are, i.e., come to an isomorphic (for finite values of $\kappa$ ) algebra $\mathcal{G}^{(\kappa)}=\kappa^{-1} \mathcal{G}_{ \pm 3} \oplus \mathcal{G}_{ \pm 2} \oplus$ $\kappa^{-1} \mathcal{G}_{ \pm 1} \oplus \mathcal{G}_{0}$; and then tend $\kappa$ to infinity in the commutation relations for the elements of $\mathcal{G}^{(\kappa)}$. . As a result we arrive at the algebra $\mathcal{G}^{(\infty)}$ which is not already simple; it is the semi-direct sum of the semisimple Lie algebra $B_{2}\left(=\left\{X_{ \pm 12}, X_{ \pm 123}, X_{ \pm 1233}, X_{ \pm 3}, h_{1}+h_{2}, h_{3}\right\}\right) \oplus g l(1)\left(=\left\{2 h_{2}+h_{3}\right\}\right)$ and the 10 dimensional commutative multiplet of the elements $R_{a}^{ \pm}, 1 \leq a \leq 5$, obtained from the elements $X_{ \pm 1}, X_{ \pm 2}, X_{ \pm 23}, X_{ \pm 233}, X_{ \pm 12233}$. With this procedure, system $(2.28)-(2.30)$ takes the form

$$
\begin{aligned}
& \partial_{+}\left(g_{0}^{-1} \partial_{-} g_{0}\right)=\left[X^{-}, g_{0}^{-1} X^{+} g_{0}\right], \\
& \partial_{ \pm} V^{\mp}=\left[X^{\mp}, g_{0}^{\mp 1} V^{ \pm} g_{0}^{ \pm 1}\right]
\end{aligned}
$$

where equation (6.17) formally looks as the standard nonabelian Toda system associated with the Lie algebra $B_{2} \oplus g l(1)$ endowed with the chosen gradation (of course, the meaning of the elements $X^{ \pm}$is different than there); while the second one describes an abelian multiplet of the fields $r_{i}^{ \pm}, 1 \leq i \leq 4$, entering the decomposition $V^{ \pm}=\sum_{i} r_{i}^{ \pm} R_{i}^{ \pm}$. In a component form these equations read as

$$
\begin{aligned}
& \partial_{+} \partial_{-} a_{1}=-4 e^{-a_{1}+a_{2}-2(\alpha+\gamma)} \operatorname{ch}^{2} \beta, \\
& \partial_{+} \partial_{-} a_{2}=-4 e^{-a_{1}+a_{2}-2(\alpha+\gamma)} \operatorname{ch}^{2} \beta, \\
& \partial_{+}\left(\partial_{-} \gamma+\operatorname{ch} 2 \beta \partial_{-} \alpha\right)=-4 e^{-a_{1}+a_{2}-2(\alpha+\gamma)} \operatorname{ch}^{2} \beta \\
& \partial_{+}\left[e^{a_{2}-2 \gamma}\left(\partial_{-} \beta+\operatorname{sh} 2 \beta \partial_{-} \alpha\right)\right]=0, \\
& \partial_{+}\left[e^{-a_{2}+2 \gamma}\left(\partial_{-} \beta-\operatorname{sh} 2 \beta \partial_{-} \alpha\right)\right]=2 e^{-a_{1}-2 \alpha} \operatorname{sh} 2 \beta ; \\
& \partial_{ \pm} r_{1}^{\mp}=\mp 4 e^{a_{1}-2(\alpha+\gamma)}\left[-\frac{1}{2} e^{4 \alpha} \operatorname{sh}^{2} \beta r_{2}^{+}-\frac{1}{2} e^{2 \alpha} \operatorname{sh} 2 \beta r_{3}^{ \pm}\right. \\
& \left.+\operatorname{ch}^{2} \beta r_{4}^{+}\right], \quad \partial_{ \pm} r_{4}^{\mp}= \pm e^{-2 a_{1}+a_{2}} r_{1}^{ \pm} \\
& \partial_{ \pm} r_{2}^{\mp}=\partial_{ \pm} r_{3}^{\mp}=0 .
\end{aligned}
$$

\footnotetext{
${ }^{9}$ Of course, this is only one of all posssible contractions of the algebra $B_{3}$.
} 
Here, by the evident reason, we omit the factor $\xi$; if it is zero, then the system is trivialised. Introducing the new functions $2 \nu \equiv a_{1}-a_{2}, 2 \sigma \equiv a_{1}+a_{2}$, one sees that $\nu$ satisfies the Laplace equation $\partial_{+} \partial_{-} \nu=0$, whose general solution is $\nu\left(z_{+}, z_{-}\right)=\nu^{+}\left(z_{+}\right)+\nu^{-}\left(z_{-}\right)$with $\nu^{ \pm}\left(z_{ \pm}\right)$being arbitrary functions of their arguments. Hence, shifting the functions $\gamma$ and $\sigma$ by $-\nu$, we have the following form of our system:

$$
\begin{aligned}
& \partial_{+} \partial_{-} \sigma=-4 e^{-2(\alpha+\gamma)} \operatorname{ch}^{2} \beta, \\
& \partial_{+}\left[\partial_{-}(\gamma-\sigma)+\operatorname{ch} 2 \beta \partial_{-} \alpha\right]=0, \\
& \partial_{+}\left[e^{\sigma-2 \gamma}\left(\partial_{-} \beta+\operatorname{sh} 2 \beta \partial_{-} \alpha\right)\right]=0, \\
& \partial_{+}\left[e^{-\sigma+2 \gamma}\left(\partial_{-} \beta-\operatorname{sh} 2 \beta \partial_{-} \alpha\right)\right]=2 e^{-\sigma-2 \alpha} \operatorname{sh} 2 \beta ; \\
& \partial_{ \pm} r_{1}^{\mp}=\mp 4 e^{\sigma-2(\alpha+\gamma)}\left[-\frac{1}{2} e^{4 \alpha} \operatorname{sh}^{2} \beta r_{2}^{ \pm}-\frac{1}{2} e^{2 \alpha} \operatorname{sh} 2 \beta r_{3}^{ \pm}\right. \\
& \left.+\operatorname{ch}^{2} \beta r_{4}^{ \pm}\right], \quad \partial_{ \pm} r_{4}^{\mp}= \pm e^{-\sigma} r_{1}^{ \pm}, \\
& \partial_{ \pm} r_{2}^{\mp}=\partial_{ \pm} r_{3}^{\mp}=0 .
\end{aligned}
$$

An effective action corresponding to subsystem (6.26) - 6.29) describing a (nontrivial) target space metric and the dilaton field, is given by the expression

$$
\begin{aligned}
\mathcal{I}_{\mathrm{eff}}^{(2)} & =\frac{1}{2} \int_{\mathcal{M}}\left\{4 \partial_{+} \alpha \partial_{-} \alpha+\partial_{+}(\sigma-2 \gamma) \partial_{-}(\sigma-2 \gamma)+\partial_{+} \sigma \partial_{-} \sigma\right. \\
& \left.+4 \partial_{+} \beta \partial_{-} \beta-4 \partial_{+}(\sigma-2 \gamma) \partial_{-} \alpha \operatorname{ch} 2 \beta+8 e^{-2(\alpha+\gamma)} \operatorname{ch}^{2} \beta\right\}
\end{aligned}
$$

With a new function $\omega$ defined by the differential relations

$$
\partial_{+} \omega=-\operatorname{sh}^{2} \beta \partial_{+}\left(\gamma-\frac{\sigma}{2}\right), \quad \partial_{-} \omega=-\operatorname{sh}^{2} \beta \partial_{-} \alpha,
$$

which are not contradictive because system $(6.26)-(\sqrt{6.29})$ provides the compatibility condition, $\partial_{+} \partial_{-} \omega=\partial_{-} \partial_{+} \omega$; one can easily get convinced that equations (6.27) - 6.29) are rewritten as

$$
\begin{aligned}
& \partial_{+} \partial_{-} \beta=\operatorname{sh} 2 \beta e^{-2(\alpha+\gamma)}+4 \frac{\operatorname{ch} \beta}{\operatorname{sh}^{3} \beta} \partial_{+} \omega \partial_{-} \omega \\
& \partial_{+}\left(\operatorname{cth}^{2} \beta \partial_{-} \omega+\frac{1}{4} \partial_{-} \sigma\right)+\partial_{-}\left(\operatorname{cth}^{2} \beta \partial_{+} \omega+\frac{1}{4} \partial_{+} \sigma\right)=0 .
\end{aligned}
$$

It seems natural to consider a subclass of the solutions to equations $(6.30)$ - (6.31) corresponding to a choice $r_{2}^{ \pm}=r_{3}^{ \pm}=0$ when this system for the 
matter fields takes the form

$$
\partial_{ \pm} r_{1}^{\mp}=\mp 4 e^{\sigma-2(\alpha+\gamma)} \operatorname{ch}^{2} \beta r_{4}^{ \pm}, \quad \partial_{ \pm} r_{4}^{\mp}= \pm e^{-\sigma} r_{1}^{ \pm} .
$$

Then, using (6.26), the first set of equations (6.36) is rewritten as

$$
\partial_{ \pm} r_{1}^{\mp}= \pm e^{\sigma} \partial_{+} \partial_{-} \sigma r_{4}^{ \pm}
$$

thereof, with account of the second set of equations (6.36), we obtain

$$
\partial_{ \pm}\left(e^{-\sigma} r_{1}^{\mp}\right)= \pm \partial_{\mp}\left(r_{4}^{ \pm} \partial_{ \pm} \sigma\right) .
$$

After that, again with the help of the second set of equations (6.36), we represent functions in the parenthesis in the 1.h.s. of 6.37) as $e^{-\sigma} r_{1}^{\mp}=$ $\mp \partial_{\mp} r_{4}^{ \pm}$, so that (6.37) is read as

$$
\partial_{\mp}\left(\partial_{ \pm} r_{4}^{ \pm}+r_{4}^{ \pm} \partial_{ \pm} \sigma\right)=0 .
$$

Hence, for the matter fields $r_{4}^{ \pm}$and $r_{1}^{ \pm}$we have the following general solutions

in terms of the function $\sigma$ and two arbitrary functions $\sigma_{0}^{ \pm}$satisfying the Laplace equation:

$$
r_{4}^{ \pm}=e^{-\sigma-\sigma_{0}^{ \pm}}, \quad r_{1}^{ \pm}=e^{\sigma} \partial_{ \pm} e^{-\sigma-\sigma_{0}^{ \pm}}
$$

The general solution to the Toda type system $(6.26)-(6.29)$ corresponding to the effective action (6.32), or the system of form (6.26), (6.34) and (6.35), is obtained directly from the general solution for equations $(2.28)-(2.30)$, with the same contraction procedure as above. It is determined by four (or, respectively, three) arbitrary functions depending on $z_{+}$and the same number of functions of $z_{-}$; and looks similar to those for the standard nonabelian Toda system [11] associated with the algebra $B_{3}$ endowed with gradation (6.1).

\section{Relation Between Abelian and Nonabelian Systems}

Of course, a given Lie algebra posseses different gradations. So, typically we may consider

$$
\mathcal{G}=\oplus_{m \in Z} \mathcal{G}_{m}^{1} \equiv \mathcal{G}_{-}^{1} \oplus \mathcal{G}_{0}^{1} \oplus \mathcal{G}_{+}^{1}=\oplus_{m \in Z} \mathcal{G}_{m}^{2} \equiv \mathcal{G}_{-}^{2} \oplus \mathcal{G}_{0}^{2} \oplus \mathcal{G}_{+}^{2} .
$$


In general, a gauge potential satisfying the grading condition with respect to the first gradation, i.e. $A_{ \pm} \in \mathcal{G}_{0}^{1} \oplus \mathcal{G}_{ \pm}^{1}$, will not satisfy it with respect to the second, i.e. $A_{ \pm} \notin \mathcal{G}_{0}^{2} \oplus \mathcal{G}_{ \pm}^{2}$. However it may be gauge equivalent to a vector potential which does. This is the case if the first gradation is of the nonabelian type - that is if $\mathcal{G}_{0}^{1} \equiv \mathcal{G}_{0}^{\text {na }}$ is non abelian, while the second is the principal gradation where $\mathcal{G}_{0}^{2} \equiv \mathcal{G}_{0}^{\mathrm{a}}$ is generated by the Cartan elements. Thus, in principle, one can relate this or that nonabelian system to an abelian system whose connection form contains the relevant number of the grading subspaces, which will be larger than one, but not necessarily maximal. In this, for the principal gradation of $\mathcal{G}, \sum_{m=1}^{m \max } \operatorname{dim} \mathcal{G}_{m}=\sum_{i} l_{i}$ where $l_{i}$ are the exponents of $\mathcal{G}$. Here the corresponding gauge transformation which, of course, changes the grading spectrum of the initial connection form, is generated by some nonabelian subalgebra of $\mathcal{G}_{0}^{\text {na }}$. In terms of the associated $W$-algebras, it means that, starting from the $W$-algebra corresponding to an appropriate number of the grading subspaces in (2.1) for the principal gradation of $\mathcal{G}$, one can obtain the $W$-algebras associated with the systems like (2.15) for other gradations, just imposing a relevant differential polynomial constraints. In terms of the associated $W$-geometries, it means that the target space corresponding to the system generated by the maximal number of the grading subspaces for the principal gradation of $\mathcal{G}$ contains all possible types of the metrics associated with nonabelian systems (2.15) and, in particular, all possible types of the black matter described by this system.

Let us give some examples related to the simple Lie algebras $B_{2}, B_{3}$ and $B_{4}$.

$B_{2}$ : Here the set $\Delta_{m}^{+}$of the positive roots corresponding to the root vectors in the subspace $\mathcal{G}_{m}$ for the principal gradation, is

$$
\Delta_{1}^{+}=\left\{\pi_{1}, \pi_{2}\right\}, \Delta_{2}^{+}=\left\{\pi_{1}+\pi_{2}\right\}, \Delta_{3}^{+}=\left\{\pi_{1}+2 \pi_{2}\right\} ;
$$

and to enlarge the (abelian) subalgebra $\mathcal{G}_{0}^{\mathrm{a}}=\left\{h_{1}, h_{2}\right\}$ to a nonabelian one, equiped by the elements $\mathcal{G}_{0}^{\text {na }}=\left\{h_{1}, h_{2}, X_{ \pm 1}\right\}$, it is enough to make a gauge transformation, generated by the elements $X_{ \pm 1}$, of the connection form (2.1) taking values in the subspaces $\mathcal{G}_{ \pm m}$ with $m=1,2$. At the same time, the analogous procedure related to $\mathcal{G}_{0}^{\text {na }}=\left\{h_{1}, h_{2}, X_{ \pm 2}\right\}$, requires to use the connection with values in the subspaces with $m=1,2,3$.

Note that here, for the principal gradation, the subalgebra $\mathcal{G}_{0}$ contains 1 element $\left(h_{1}\right)$ commuting with $\mathcal{G}_{3}$, and 1 element $\left(h_{2}\right)$ commuting with $\mathcal{G}_{2}$. 
$B_{3}$ : Here the set $\Delta_{m}^{+}$for the principal gradation, is

$$
\begin{gathered}
\Delta_{1}^{+}=\left\{\pi_{1}, \pi_{2}, \pi_{3}\right\}, \Delta_{2}^{+}=\left\{\pi_{1}+\pi_{2}, \pi_{2}+\pi_{3}\right\}, \Delta_{3}^{+}=\left\{\pi_{1}+\pi_{2}+\pi_{3}, \pi_{2}+2 \pi_{3}\right\} ; \\
\Delta_{4}^{+}=\left\{\pi_{1}+\pi_{2}+2 \pi_{3}\right\} ; \Delta_{5}^{+}=\left\{\pi_{1}+2 \pi_{2}+2 \pi_{3}\right\} ;
\end{gathered}
$$

and to enlarge the (abelian) subalgebra $\mathcal{G}_{0}^{\mathrm{a}}=\left\{h_{1}, h_{2}, h_{3}\right\}$ to a nonabelian one, equiped by the elements $\mathcal{G}_{0}^{\text {na }}=\left\{h_{1}, h_{2}, h_{3}, X_{ \pm 1}\right\}$, it is enough to make a gauge transformation, generated by the elements $X_{ \pm 1}$, of the connection form (2.1) taking values in the subspaces $\mathcal{G}_{ \pm m}$ with $m=1,2,3$. The analogous procedure related to $\mathcal{G}_{0}^{\text {na }}=\left\{h_{1}, h_{2}, h_{3}, X_{ \pm 2}\right\}$, requires to use the connection with values in the subspaces with $m=1,2,3,4,5$; and those for $\mathcal{G}_{0}^{\text {na }}=$ $\left\{h_{1}, h_{2}, h_{3}, X_{ \pm 3}\right\}$, requires the connection with values in the subspaces with $m=1,2,3,4$.

Here, for the principal gradation, the subalgebra $\mathcal{G}_{0}$ contains 2 elements $\left(h_{1}, h_{3}\right)$ commuting with $\mathcal{G}_{5} ; 2$ elements $\left(h_{1}+h_{2}, 2 h_{2}+h_{3}\right)$ commuting with $\mathcal{G}_{4} ; 1$ element $\left(h_{2}\right)$ commuting with $\mathcal{G}_{3}$; and 1 element $\left(h_{1}+h_{2}+h_{3}\right)$ commuting with $\mathcal{G}_{2}$.

$B_{4}$ : Here the set $\Delta_{m}^{+}$for the principal gradation, is

$$
\begin{gathered}
\Delta_{1}^{+}=\left\{\pi_{1}, \pi_{2}, \pi_{3}, \pi_{4}\right\}, \Delta_{2}^{+}=\left\{\pi_{1}+\pi_{2}, \pi_{2}+\pi_{3}, \pi_{3}+\pi_{4}\right\} ; \\
\Delta_{3}^{+}=\left\{\pi_{1}+\pi_{2}+\pi_{3}, \pi_{2}+\pi_{3}+\pi_{4}, \pi_{3}+2 \pi_{4}\right\}, \\
\Delta_{4}^{+}=\left\{\pi_{1}+\pi_{2}+\pi_{3}+\pi_{4}, \pi_{2}+\pi_{3}+2 \pi_{4}\right\} ; \\
\Delta_{5}^{+}=\left\{\pi_{1}+\pi_{2}+\pi_{3}+2 \pi_{4}, \pi_{2}+2 \pi_{3}+2 \pi_{4}\right\} ; \\
\Delta_{6}^{+}=\left\{\pi_{1}+\pi_{2}+2 \pi_{3}+2 \pi_{4}\right\} ; \Delta_{7}^{+}=\left\{\pi_{1}+2 \pi_{2}+2 \pi_{3}+2 \pi_{4}\right\} .
\end{gathered}
$$

Here the subalgebra $\mathcal{G}_{0}$ contains 3 elements $\left(h_{1}, h_{3}, h_{4}\right)$ commuting with $\mathcal{G}_{7} ; 3$ elements $\left(h_{1}-h_{3}, h_{2}+h_{3}, h_{4}\right)$ commuting with $\mathcal{G}_{6} ; 2$ elements $\left(h_{2}, h_{1}+\right.$ $h_{3}$ ) commuting with $\mathcal{G}_{5} ; 2$ elements $\left(h_{2}+h_{3}, 2 h_{3}+h_{4}\right)$ commuting with $\mathcal{G}_{4}$; 1 element $\left(2 h_{1}+2 h_{2}+h_{4}\right)$ commuting with $\mathcal{G}_{3}$; and 1 element $\left(h_{2}+h_{3}+h_{4}\right)$ commuting with $\mathcal{G}_{2}$.

To clarify better the picture, give as an illustration a component form of equations (2.15) which are obtained by the gauge transformation generated by the elements $X_{ \pm r}$ from those for the principal gradation of the algebra $B_{r}$ 
with account of its higher grading subspaces. The arising system looks as follows [11]:

$$
\begin{aligned}
& \partial_{+} \partial_{-} x_{i}=e^{(k x)_{i}}-\left(\delta_{i, r-1}-\delta_{i, r}\right) \frac{\operatorname{sh} \frac{x_{r-1}-x_{r}}{2}}{\operatorname{ch} \frac{3 x_{r-1}-x_{r}}{2}} \partial_{+} x_{r+1} \partial_{-} x_{r+1} ; \\
& \partial_{+} \partial_{-} x_{r+1}=-\frac{\partial_{-}\left(x_{r-1}-x_{r}\right) \partial_{+} x_{r+1}+\partial_{+}\left(x_{r-1}-x_{r}\right) \partial_{-} x_{r+1}}{\operatorname{sh}\left(x_{r-1}-x_{r}\right)}
\end{aligned}
$$

where $k$ is the Cartan matrix of the subalgebra $D_{r} \in B_{r}, 1 \leq i \leq r$; and possesses the Lagrangian function

$$
L=\frac{1}{2}\left(\sum_{i, j=1}^{r} k_{i j} \partial_{+} x_{i} \partial_{-} x_{j}-4 \text { th } \frac{{ }^{2}}{x_{r-1}-x_{r}} \partial_{+} x_{r+1} \partial_{-} x_{r+1}\right)+\sum_{i=1}^{r} e^{(k x)_{i}} .
$$

Note that just this nontrivial form of the metric in the target space causes an origin of a black matter phenomena in the spirit of Witten, while obtained from nonabelian Toda system, see [1]. As it was also mentioned in that paper by an example of $B_{2}$, the structure of the $W$-algebra elements here is highly nontrivial. In particular, one can get convinced that the form of three most important $W$-elements of the second order, written before in [24] only for the case of $B_{2}$, is

$$
\begin{aligned}
& { }^{0} W_{2}^{ \pm}=\sum_{i=1}^{r} \partial_{ \pm}^{2} x_{i}-\frac{1}{2} \sum_{i, j=1}^{r} k_{i j} \partial_{ \pm} x_{i} \partial_{ \pm} x_{j}+2 \text { th } \frac{}{2} \frac{R}{2}\left(\partial_{ \pm} x_{r+1}\right)^{2} \\
& { }^{ \pm} W_{2}^{ \pm}=e^{\Phi} \partial_{ \pm}\left[e^{ \pm \Omega-\Phi}\left(\partial_{ \pm} R \pm 2 \text { th } \frac{R}{2} \partial_{ \pm} x_{r+1}\right)\right]+e^{ \pm \Omega} \partial_{ \pm} x_{r-2} \partial_{ \pm} R
\end{aligned}
$$

where $R \equiv x_{r-1}-x_{r}, \Phi \equiv x_{r-1}+x_{r}$; and the function $\Omega$ satisfies the relations

$$
\partial_{+} \Omega=\frac{\operatorname{ch} R}{\operatorname{ch}^{2} \frac{R}{2}} \partial_{+} x_{r+1}, \quad \partial_{-} \Omega=\frac{1}{\operatorname{ch}^{2} \frac{R}{2}} \partial_{-} x_{r+1} .
$$

Having expressions for the $W$-elements, one can study the problem of the integrability of a system on a half-line $r \equiv z_{+}-z_{-}=0$. Probably, the simplest way to do that is to equate the corresponding $W_{m}^{ \pm}$-elements, $\partial_{\mp} W_{m}^{ \pm}=0$, at $z_{+}=z_{-}$. For the special case of the Liouville equation it was shown in 25 that its integrability respects the boundary condition

$$
\partial_{r} x=\lambda \cdot e^{x} \quad \text { at } r=0
$$


for an arbitrary constant $\lambda$. An analogous situation takes place also for the sine (or sinh)-Gordon model, see references given in [26] addressed to a more general situation with the (abelian) affine Toda field theory associated with an affine Lie Kac-Moody algebra $\tilde{\mathcal{G}}$. Here, except for the $A_{1}^{(1)}$, i.e., the sineGordon model, for the simply laced algebras there are only two possibilities for constants $\lambda_{i}$ entering the boundary conditions like (7.7) written for the fields $x_{i}, 1 \leq i \leq r$; namely $\left|\lambda_{i}\right|=2$, or all $\lambda_{i}=0$. One can easily show that for the corresponding finite case the situation is the same. It is interesting to note that, in the same way as for the abelian Toda systems associated with non simply laced Lie algebras, nonabelian Toda systems [16] in general seemingly do not obey such rigid restrictions on these constants.

Let us show it by the example of $B_{2}$ where the boundary conditions contain one arbitrary constant. Equating the corresponding $W^{ \pm}$-elements (7.4), (7.5), all three of them here being of the 2nd order, one has from the expressions for ${ }^{0} W_{2}^{ \pm}$that

$$
\partial_{r} x_{i}=\lambda_{i} \cdot e^{x_{i}} \quad \text { at } r=0, i=1,2
$$

where $\lambda_{i}$ are arbitrary constants yet; while ${ }^{ \pm} W_{2}^{ \pm}$give more rigid conditions that $\lambda_{1}=\lambda_{2}$, and $x_{3}=\partial_{r} x_{3}=0$ at $r=0$. So, finally we have the following, in fact, sufficient boundary conditions:

$$
\partial_{r} x_{i}=\lambda \cdot e^{x_{i}} ; i=1,2 ; x_{3}=\partial_{r} x_{3}=0 \quad \text { at } r=0 .
$$

\section{A Bäcklund Type Transformation}

In this section we discuss a Bäcklund type transformation for the systems under consideration, and a construction of the corresponding $W$-elements by an example of equations (2.37) - (2.39), following the line of those in [27], [24], see also [11]. Let us begin with the connection components of form (4.8); rewrite them for the case of the series $A_{r}$ endowed with the principal gradation, and take $M=r$. Moreover, supply the terms $X^{ \pm}$with the factor $\xi$, so to have a possibility of a direct reduction to the case of the standard Toda system. Parametrising the functions $g_{0}$ and $V_{m}^{ \pm}$as in the section 2, i.e., in our case

$$
g_{0}=e^{-\sum_{j=1}^{r} x_{j} h_{j}}, \quad V_{m}^{ \pm}=\sum_{p=1}^{r-m+1} e^{-\sum_{j=p}^{m+p} \rho_{j}} \partial_{ \pm} f_{p}^{ \pm m} X_{ \pm \sum_{i=p}^{m+p} \pi_{i}} ;
$$


we have

$$
\begin{aligned}
A_{+} & =\sum_{m=1}^{r-1} \sum_{p=1}^{r-m+1} \partial_{+} f_{p}^{+m} X_{+\sum_{i=p}^{m+p-1} \pi_{i}}+\xi e^{\sum_{j=1}^{r} \rho_{j}} X_{+\sum_{i=1}^{r} \pi_{i}} \\
A_{-} & =-\sum_{i=1}^{r} \partial_{-} x_{i} h_{i}+\sum_{m=1}^{r-1} \sum_{p=1}^{r-m+1} e^{-\sum_{j=p}^{m+p-1} \rho_{j}} \partial_{-} f_{p}^{-m} X_{-\sum_{i=p}^{m+p-1} \pi_{i}} \\
& +\xi X_{-} \sum_{i=1}^{r} \pi_{i} .
\end{aligned}
$$

Introduce the functions $\Psi_{a}^{A}\left(z_{+}, z_{-}\right) \equiv<A|g| a>$, with $\mid a>$ being the basis vectors of an $N$ dimensional irreducible representation of $A_{r}$ with the highest weight components $l_{i}, 1 \leq i \leq r$, and $<A \mid$ - those of the dual one; and recall that the connection components $A_{ \pm}$are expressed in terms of $g$ as (4.1). Since we deal here with the principal gradation, it is convenient to use the Verma type basis for the representation space, in other words

$$
|a>\equiv| j_{1}, \cdots, j_{a}>=X_{-j_{a}} \cdots X_{-j_{1}} \mid 0>_{l}, 1 \leq j \leq r, 0 \leq a \leq N-1
$$

$\left|j_{1}, \cdots, j_{0}>\equiv\right| 0>_{l}$; where $\mid 0>_{l}$ is the highest weight vector. Thanks to the defining relation (2.31), there take place the following evident relations:

$$
\begin{aligned}
& X_{-j}\left|j_{1}, \cdots, j_{a}>_{l}=\right| j_{1}, \cdots, j_{a}, j>_{l}, \\
& h_{j}\left|j_{1}, \cdots, j_{a}>_{l}=\left(l_{j}-\sum_{b=1}^{a} k_{j_{b} j}\right)\right| j_{1}, \cdots, j_{a}>_{l}, \\
& X_{+j}\left|j_{1}, \cdots, j_{a}>_{l}=\sum_{b=1}^{a} \delta_{j j_{b}}\left(l_{j}-\sum_{c=1}^{b-1} k_{j_{c} j}\right)\right| j_{1}, \cdots, j_{a}>_{l}^{(b)} ;
\end{aligned}
$$

where the superscript at the vector $\mid j_{1}, \cdots, j_{a}>_{l}^{(b)}$ means that here, in the product $X_{-j_{a}} \cdots X_{-j_{1}}$, the root vector $X_{-j_{b}}$ is absent. In fact, since we are going to consider the 1 st fundamental representation of $A_{r}, j_{a}=a, l_{j}=\delta_{1 j}$, the last formula has the form

$$
X_{+j}\left|a>=\delta_{j a}\left(\delta_{1 j}-\sum_{c=1}^{a-1} k_{c j}\right)\right| a-1>;
$$

here and in what follows the subscript $l$ will be omitted.

Differentiating the functions $\left\langle A|g| a>\right.$ over $z_{+}$and over $z_{-}$with account of (4.1), i.e., $\partial_{ \pm} g=g A_{ \pm}$, and using (8.1), (8.2) and (8.3), after some simple 
algebra, we arrive at the equations of the 1st order for these functions,

$$
\begin{aligned}
& \partial_{+} \Psi_{a}^{A}=\left(\partial_{+} f_{1}^{+a}+\delta_{a r} \xi e^{\sum_{i=1}^{r} \rho_{i}}\right) \Psi_{0}^{A}+\sum_{m=1}^{a-1} \partial_{+} f_{a-m+1}^{+m} \Psi_{a-m}^{A}, \\
& \partial_{-}\left(e^{x_{1}-\sum_{b=1}^{a} \rho_{b}} \Psi_{a}^{A}\right)=e^{x_{1}-\sum_{b=1}^{a} \rho_{b}}\left[\delta_{a 0} \xi \Psi_{r}^{A}+\right. \\
& \left.\sum_{m=1}^{\min (r-a, r-1)} e^{-\sum_{i=a+1}^{m+a} \rho_{i}} \partial_{-} f_{a+1}^{-m} \Psi_{a+m}^{A}\right] .
\end{aligned}
$$

With the notation $\Phi_{a}^{A} \equiv e^{x_{1}-\sum_{b=1}^{a} \rho_{b}} \Psi_{a}^{A}$, the last system (8.5) is simplified as

$$
\left.\partial_{-} \Phi_{a}^{A}=\delta_{a 0} \xi e^{\sum_{i=1}^{r} \rho_{i}} \Phi_{r}^{A}+\sum_{m=1}^{\min (r-a, r-1)} \partial_{-} f_{a+1}^{-m} \Phi_{a+m}^{A}\right] .
$$

Recall that here $0 \leq a \leq r$; and $f_{1}^{0}=f_{1}^{r} \equiv 0$ by definition.

Now one can easily verify that differentiation of system (8.4) over $z_{-}$and those of system (8.5) over $z_{+}$gives equations (2.37) - (2.39), so that (8.4) and (8.5) realise a Bäcklund type transformation for these equations. From the other hand, with a minor re-notation, equations (8.4) and (8.5) are equivalent to the Frenet-Serret formulas for the moving frame [18]; while for other simple Lie algebras we expect the similar situation with respect to the $W$ surfaces introduced in [19]. Due to their nilpotent structure, equations (8.4) and (8.5) can be explicitly solved in the same way as it is given in [31]; their general solution is expressed in terms of arbitrary functions $\Psi_{0}^{A}\left(z_{-}\right), \Phi_{r}^{A}\left(z_{+}\right)$ and the known solution for system (2.37) - (2.39). In particular, the last step in the integration scheme gives the equation of the $(r+1)$ th order for the functions $\Psi_{0}^{A}$, which establishes a relation between its coefficients, in fact the $W$-potentials or conserved currents, and the set of linearly independent fundamental solutions $\Psi_{0}^{A}\left(z_{-}\right)$. Note that such $W$-elements, realised as differential polynomials in fields $x_{i}$ and $f_{i}^{ \pm m}$, are nothing but the characteristic integrals; they provide the integrability property of equations $(2.37)-(2.39)$ in the same way as the corresponding characteristic integrals for the standard Toda systems, see e.g., [28], [27], [29], [11]. To write down the $W$-elements explicitly, let us derive for them the generating equation, using e.g., system (8.6). Since this equation is an ordinary differential equation for the function $\Psi_{0} \equiv e^{-x_{1}} \Phi_{0}$ which does not depend on the variable $z_{+}$thanks to the 1 st equation in (8.4) for $a=0$, the procedure for obtaining the equation consists 
in a consequent elimination of the functions $\Phi_{a}^{A}$ with $a=1, a=2$, etc., up to the last one satisfying $\partial_{-} \Phi_{r}^{A}=0$.

One can easily get convinced from (8.6) that

$$
\sum_{a=p}^{r} u_{a} \partial_{-} \Phi_{a}^{A}=\sum_{a=p+1}^{r}\left(\sum_{m=p}^{a-1} u_{m} \partial_{-} f_{m+1}^{-(a-m)}\right) \Phi_{a}^{A}
$$

with some arbitrary functions $u_{a}$; and hence

$$
\sum_{a=p}^{r} \partial_{-}\left(u_{a} \Phi_{a}^{A}\right)=\sum_{a=p+1}^{r}\left(\sum_{m=p}^{a-1} u_{m} \partial_{-} f_{m+1}^{-(a-m)}+\partial_{-} u_{a}\right) \Phi_{a}^{A}+\partial_{-} u_{p} \Phi_{p}^{A} .
$$

With account of (8.8) and equations (8.6) we obtain the recurrent relations

$$
\begin{aligned}
& \partial_{-}\left(\frac{1}{v_{s}^{(s)}} \partial_{-}\left(\frac{1}{v_{s-1}^{(s-1)}} \partial_{-}\left(\cdots \partial_{-}\left(\frac{1}{v_{2}^{(2)}} \partial_{-}\left(\frac{1}{v_{1}^{(1)}} \partial_{-} \Phi_{0}^{A}\right)\right) \cdots\right)\right)\right) \\
& =v_{s+1}^{(s+1)} \Phi_{s+1}^{A}+\sum_{a=s+2}^{r} v_{a}^{(s+1)} \Phi_{a}^{A}
\end{aligned}
$$

where

$$
\begin{aligned}
v_{a}^{(n)} & =\sum_{m=n}^{a-1} \frac{v_{m}^{(n-1)}}{v_{n-1}^{(n-1)}} \partial_{-} f_{m+1}^{-(a-m)}+\partial_{-}\left(\frac{v_{a}^{(n-1)}}{v_{n-1}^{(n-1)}}\right)+\partial_{-} f_{n}^{-(a-n+1)}, \quad n \leq a \leq r ; \\
v_{a}^{(1)} & =\partial_{-} f_{1}^{-a}+\xi \delta_{a r} e^{\sum_{i=1}^{r} \rho_{i}}, \quad 1 \leq a \leq r ;
\end{aligned}
$$

and recall once more that $f_{1}^{-r} \equiv 0$. Finally, one comes to the following generating equation for the $W$-elements:

$$
\partial_{-}\left(\frac{1}{v_{r}^{(r)}} \partial_{-}\left(\frac{1}{v_{r-1}^{(r-1)}} \partial_{-}\left(\cdots \partial_{-}\left(\frac{1}{v_{2}^{(2)}} \partial_{-}\left(\frac{1}{v_{1}^{(1)}} \partial_{-} \Phi_{0}^{A}\right)\right) \cdots\right)\right)\right)=0,
$$

from which the corresponding $W$-elements are constructed in accordance with the formula

$$
\sum_{m=0}^{r} W_{m}^{-} \partial_{-}^{r-m+1} \Phi_{0}^{A}=0
$$

An analogous representation is easily obtained for the elements $W_{m}^{+}, \partial_{-} W_{m}^{+}=$ 0 , starting from system (8.4). 
Here two comments are in time; the first, equation (8.11) should be understood up to singularity lines related to zeros of the denominators $v_{a}^{(a)}$; the second, since not all the fields, contained in $g_{0}$ and $V_{m}^{ \pm}$, are the primary ones, the complete set of the $W$-elements corresponding to system (2.28) $(2.30)$ or $(2.37)-(2.39)$ is, in general, different than those for the equations satisfied by the primary fields. Of course, these comments are relevant for nonabelian cases of the standard Toda systems (2.15) as well. Note also that for the case of the standard abelian $A_{r}$ Toda system (2.32), equation (8.11) coincides with those in [27].

\section{Outlook}

Finishing up this paper, we would like to formulate forthcoming problems which seem to be very interesting and important, and can be solved on the base of the results obtained here. First, we have not systematically studied the $\mathrm{W}$-symmetries of our systems, but we already derived the conserved $W$ charges for the case of $A_{r}$ in section 8 . In our extended scheme, one may encounter interesting generalisations of the $\mathrm{W}$-algebras that already arose from the standard Toda theories. Second, introducing matter in the non abelian Toda case is a step towards a concrete description of the black hole formation from collapsing matter and evaporation, using integrable sytem of equations. Examples of the corresponding field equations were given in section 6 . Third, it will be interesting to study configurations of the Toda fields coupled to matter fields, with boundaries for abelian and nonabelian configurations, as was begun in section 7 . Fourth, the considerations given in the present paper can be extended $\square$ in a rather direct way for the case of the systems generated by the higher grading subspaces of the affine Lie algebras, so to have a generalisation of the abelian and nonabelian affine (conformally affine) equations and in turn solitons in a black and field matter system; and also for supersymmetric systems based on the corresponding superalgebras. Last, from the differential geometry point of view it would be very nice to describe, in the spirit of [18], [19], the $W$-geometries and an analogue of the Plücker embedding problem in terms of the solutions to the new systems discussed in the paper. Moreover, we think that it is possible to realise the integration scheme in terms of some holomorphic and antiholomorphic

\footnotetext{
${ }^{10}$ This will be done in a forthcoming article [32].
} 
distributions on the flag manifolds associated with the relevant parabolic subalgebras of the algebra $\mathcal{G}$ in question, in the same way as it has been done for the standard Toda system (2.15) in [30].

\section{Acknowledgements}

One of the authors (M. S.) wishes to acknowledge the warm hospitality and creative scientific atmosphere of the Laboratoire de Physique Théorique de l'École Normale Supérieure de Paris. This work was partially supported by the Russian Fund for Fundamental Research and International Science Foundation.

\section{References}

[1] J.-L. Gervais and M. V. Saveliev, Phys. Lett. B286 (1992), 271.

[2] A. Bilal, Nucl. Phys. B422 (1994), 258; Letters in Math. Phys. 32 (1994), 103.

[3] E. Witten, it Phys. Rev. D44 (1991), 314.

[4] G. Mandal, A. Sengupta and S. Wadia, Mod. Phys. Lett. A6 (1991), 1685.

[5] J. Harvey and A. Strominger, in TASI'92, From Black Holes and Strings to Particles, (World Scientific, Singapore, 1993).

[6] A. Strominger, Les Houches lectures on black holes, hep-th/9501071.

[7] S. P. de Alwis, Phys. Rev. 46D (1992), 5429.

[8] A. Bilal and C. G. Callan, Nucl. Phys. (1993), 73.

[9] J. G. Russo, L. Susskind and L. Thorlacius, Phys. Rev. 46D (1993), 3444; 47D (1993), 533.

[10] A. N. Leznov, Exactly integrable systems with fermionic fields, Preprint IHEP 83-7 (Serpukhov, 1983). 
[11] A. N. Leznov and M. V. Saveliev, Group-Theoretical Methods for Integration of Nonlinear Dynamical Systems, Progress in Physics Series, v. 15, Birkhaüser-Verlag, Basel, 1992.

[12] L. Fehér, L. O'Raifeartaigh, P. Ruelle, I. Tsutsui and A. Wipf, Phys. Reports, 222 1992, 1.

[13] J.-L. Gervais, L. O'Raifeartaigh, A. V. Razumov and M. V. Saveliev, Phys. Lett. 301B (1993), 41.

[14] D. A. Leites, M. V. Saveliev and V. V. Serganova, in Group Theoretical Methods in Physics, eds. V. I. Manko and M. A. Markov, v. 1 (VNU Science Press, Haarlem, 1986), 255.

[15] M. V. Saveliev, On some integrable generalisations of the continuous Toda system, Preprint LPTENS-95/17 (Paris, 1995), hep-th/9504131; to appear in J. Theor\&Math. Phys.

[16] P. Bowcock, E. Corrigan, P. E. Dorey, R. H. Rietdijk, Nucl. Phys. 445B (1995), 469.

[17] A. Bilal and J.-L. Gervais, Nucl. Phys. 314B (1989), 646; 318B (1989), 579 .

[18] J.-L. Gervais and Y. Matsuo, Comm. Math. Phys. 152 (1993), 317-368.

[19] J.-L. Gervais and M. V. Saveliev, W-Geometry of the Toda Systems Associated with Non-Exceptional Simple Lie Algebras, Preprint LPTENS93/47 (Paris, 1993), hep-th/9312040; to appear in Comm. Math. Phys.

[20] L. Ferreira, J. Miramontes, J. Guillén, Solitons, Tau-functions and Hamiltonian reduction for non-abelian conformal affine Toda theories, Preprint hep-th/9412127.

[21] N. N. Bogoliubov and D. V. Shirkov, Introduction to the Theory of Quantized Fields, Interscience, New York, 1959.

[22] R. S. Strichartz, J. Funct. Anal. 72 (1987), 320.

[23] V.G. Drinfeld and V.V. Sokolov, Journ. Sov. Math. 30 (1985), 1975.

[24] M. V. Saveliev, Mod. Phys. Lett. A5 (1990), 2223. 
[25] J.-L. Gervais and A. Neveu, Nucl. Phys. B199 (1982), 59.

[26] E. Corrigan, P. E. Dorey, R. H. Rietdijk and R. Sasaki, Phys. Lett. B333 (1994), 83.

[27] A. N. Leznov, Lett. Math. Phys. 8 (1984), 5; Funct. Anal. Appl. 18 (1984), 83; Sov. J. Theor. Math. Phys. 58 (1984), 156; in Nonlinear Processes in Physics and Turbulence, ed. R. Z. Sagdeev (Gordon and Breach, New York, 1984), 443.

[28] A. N. Leznov, V. G. Smirnov and A. B. Shabat, Sov. J. Theor. Math. Physics, 51 (1982), 10.

[29] A. V. Mikhailov, A. B. Shabat and R. I. Yamilov, Russian Math. Surveys, 42 (1987), 3.

[30] A. V. Razumov and M. V. Saveliev, Comm. Anal\&Geometry, 2 (1994), 461.

[31] J.-L. Gervais and M. V. Saveliev, Lotka-Volterra type equations and their explicit integration, Lett. Math. Phys. 34 (1995), 177.

[32] L. A. Ferreira, J.-L. Gervais, J. S. Guillen, M. V. Saveliev, Affine Toda systems coupled to matter fields, to be published. 